\title{
Integer Sequences for the Sum of Powers of Trigonometric Values
}

\author{
Kai Wang \\ Henderson, NV, USA \\ Email: kaiwang1945@gmail.com
}

How to cite this paper: Wang, K. (2019) Integer Sequences for the Sum of Powers of Trigonometric Values. Open Access Library Journal, 6: e5417.

https://doi.org/10.4236/oalib.1105417

Received: April 22, 2019

Accepted: May 28, 2019

Published: May 31, 2019

Copyright $\odot 2019$ by author(s) and Open Access Library Inc.

This work is licensed under the Creative Commons Attribution International License (CC BY 4.0).

http://creativecommons.org/licenses/by/4.0/

\begin{abstract}
We will develop some methods to generate integer sequences whose terms are sums of mixed powers of trigonometric values at angles of a heptagonal triangle. Our method will produce many new integer sequences and reproduce some sequences which are discovered using different methods by other researchers.
\end{abstract}

\section{Subject Areas}

Mathematical Analysis

\section{Keywords}

Integer Sequence, Heptagonal Triangle, Sine, Cosine, Tangent, Recurrence Equation

\section{Introduction}

Integer sequences whose terms are mixed power sums of trigonometric functions at heptagonal triangles have been studied by many researchers such as [1]. We became interested in integer sequences through the study of sums of mixed powers of trigonometric values. In [2] as corollaries of theta function identities from Ramanujans notebooks, Berndt and Zhang discovered some trigonometric identities which have zero values or integer values. We subsequently developed some methods to evaluate sums of mixed powers of trigonometric values in an unpublished paper [3]. During our studies, we also found the following integer sequence:

Theorem 1. Let

$$
t(n)=\left(\frac{\tan \frac{2 \pi}{7}}{\tan \frac{4 \pi}{7}}\right)^{n}+\left(\frac{\tan \frac{4 \pi}{7}}{\tan \frac{8 \pi}{7}}\right)^{n}+\left(\frac{\tan \frac{8 \pi}{7}}{\tan \frac{2 \pi}{7}}\right)^{n} .
$$




\section{Then}

$$
\{t(n)=3,-9,83,-753,6851,-62329,567059, \cdots \mid n=0,1,2, \cdots\},[\underline{\text { A274032] }}
$$

and

$$
\{t(-n)=3,-1,19,-25,195,-401,2131,-5545, \cdots \mid n=0,1,2, \cdots\} \quad[\underline{\text { A274075 }}]
$$

together is a two-sided integer sequence.

Since then, we have generated more integer sequences using similar methods. The purpose of this paper is to further advance our methods in a more general setting so that it has many of our previous sequences as special cases. In this paper, for convenience, Let $\theta=\frac{\pi}{7}$. Our method is based on the following proposition.

Proposition 2. Let $\{X, Y, Z\}$ be the roots of a cubic equation:

$$
t^{3}+a t^{2}+b t+c=0
$$

where $a, b, c$ are integers. Then

$$
\left\{p(n)=X^{n}+Y^{n}+Z^{n} \mid n=0,1,2, \cdots\right\}
$$

is an integer sequence with the recurrence relation:

$$
p(n)=-a p(n-1)-b p(n-2)-c p(n-3) .
$$

Our main results are the following:

Theorem 3. For integers $i, j$,

$$
\begin{gathered}
\left\{\left(\frac{\sin ^{i+j} 2 \theta}{\sin ^{i} 4 \theta \sin ^{j} 8 \theta}\right)^{n}+\left(\frac{\sin ^{i+j} 4 \theta}{\sin ^{i} 8 \theta \sin ^{j} 2 \theta}\right)^{n}+\left(\frac{\sin ^{i+j} 8 \theta}{\sin ^{i} 2 \theta \sin ^{j} 4 \theta}\right)^{n} \mid n=0, \pm 1, \pm 2, \cdots\right\}, \\
\left\{\left(\frac{\cos ^{i+j} 2 \theta}{\cos ^{i} 4 \theta \cos ^{j} 8 \theta}\right)^{n}+\left(\frac{\cos ^{i+j} 4 \theta}{\cos ^{i} 8 \theta \cos ^{j} 2 \theta}\right)^{n}+\left(\frac{\cos ^{i+j} 8 \theta}{\cos ^{i} 2 \theta \cos ^{j} 4 \theta}\right)^{n} \mid n=0, \pm 1, \pm 2, \cdots\right\}, \\
\left\{\left(\frac{\tan ^{i+j} 2 \theta}{\tan ^{i} 4 \theta \tan ^{j} 8 \theta}\right)^{n}+\left(\frac{\tan ^{i+j} 4 \theta}{\tan ^{i} 8 \theta \tan ^{j} 2 \theta}\right)^{n}+\left(\frac{\tan ^{i+j} 8 \theta}{\tan ^{i} 2 \theta \tan ^{j} 4 \theta}\right)^{n} \mid n=0, \pm 1, \pm 2, \cdots\right\}
\end{gathered}
$$

are two-sided integer sequences.

$$
\begin{aligned}
& \left\{\left(\frac{2 \sqrt{7} \sin ^{i+j+1} 2 \theta}{\sin ^{i} 4 \theta \sin ^{j} 8 \theta}\right)^{n}+\left(\frac{2 \sqrt{7} \sin ^{i+j+1} 4 \theta}{\sin ^{i} 8 \theta \sin ^{j} 2 \theta}\right)^{n}+\left(\frac{2 \sqrt{7} \sin ^{i+j+1} 8 \theta}{\sin ^{i} 2 \theta \sin ^{j} 4 \theta}\right)^{n} \mid n=0,1,2, \cdots\right\}, \\
& \left\{\left(\frac{\sqrt{7} \sin ^{i+j-1} 2 \theta}{2 \sin ^{i} 4 \theta \sin ^{j} 8 \theta}\right)^{n}+\left(\frac{\sqrt{7} \sin ^{i+j-1} 4 \theta}{2 \sin ^{i} 8 \theta \sin ^{j} 2 \theta}\right)^{n}+\left(\frac{\sqrt{7} \sin ^{i+j-1} 8 \theta}{2 \sin ^{i} 2 \theta \sin ^{j} 4 \theta}\right)^{n} \mid n=0,1,2, \cdots\right\}, \\
& \left\{\left(\frac{2 \cos ^{i+j+1} 2 \theta}{\cos ^{i} 4 \theta \cos ^{j} 8 \theta}\right)^{n}+\left(\frac{2 \cos ^{i+j+1} 4 \theta}{\cos ^{i} 8 \theta \cos ^{j} 2 \theta}\right)^{n}+\left(\frac{2 \cos ^{i+j+1} 8 \theta}{\cos ^{i} 2 \theta \cos ^{j} 4 \theta}\right)^{n} \mid n=0,1,2, \cdots\right\}, \\
& \left\{\left(\frac{\cos ^{i+j-1} 2 \theta}{\cos ^{i} 4 \theta \cos ^{j} 8 \theta}\right)^{n}+\left(\frac{\cos ^{i+j-1} 4 \theta}{\cos ^{i} 8 \theta \cos ^{j} 2 \theta}\right)^{n}+\left(\frac{\cos ^{i+j-1} 8 \theta}{\cos ^{i} 2 \theta \cos ^{j} 4 \theta}\right)^{n} \mid n=0,1,2, \cdots\right\}, \\
& \left\{\left(\frac{\sqrt{7} \tan ^{i+j+1} 2 \theta}{\tan ^{i} 4 \theta \tan ^{j} 8 \theta}\right)^{n}+\left(\frac{\sqrt{7} \tan ^{i+j+1} 4 \theta}{\tan ^{i} 8 \theta \tan ^{j} 2 \theta}\right)^{n}+\left(\frac{\sqrt{7} \tan ^{i+j+1} 8 \theta}{\tan ^{i} 2 \theta \tan ^{j} 4 \theta}\right)^{n} \mid n=0,1,2, \cdots\right\},
\end{aligned}
$$




$$
\left\{\left(\frac{\sqrt{7} \tan ^{i+j-1} 2 \theta}{\tan ^{i} 4 \theta \tan ^{j} 8 \theta}\right)^{n}+\left(\frac{\sqrt{7} \tan ^{i+j-1} 4 \theta}{\tan ^{i} 8 \theta \tan ^{j} 2 \theta}\right)^{n}+\left(\frac{\sqrt{7} \tan ^{i+j-1} 8 \theta}{\tan ^{i} 2 \theta \tan ^{j} 4 \theta}\right)^{n} \mid n=0,1,2, \cdots\right\}
$$

are integer sequences.

Note the Propositions 2 can be proven easily by induction. Theorem 3 will be proved later separately as Theorem 23 , Theorem 30 , Theorem 36 , Theorem 51, Theorem 58, Theorem 64, Theorem 79, Theorem 86, and Theorem 92.

Note that in this paper all the sequences are generated by computer.

\section{Sums of Powers of Roots for an Integer Equation}

Definition 4. Let $A, B, C$ are three nonzero numbers. For an expression $f(A, B, C)$, let

$$
\begin{gathered}
\prod f(A, B, C)=f(A, B, C) f(B, C, A) f(C, A, B) . \\
\sum f(A, B, C)=f(A, B, C)+f(B, C, A)+f(C, A, B), \\
P=A B C, Q=\sum \frac{A}{B}, R=\sum \frac{A}{C} .
\end{gathered}
$$

For a constant $K$ and integers $i, j$, let

1) $\alpha(i, j)=\alpha(i, j \mid A, B, C)=\sum \frac{A^{i+j}}{B^{i} C^{j}}$,

2) $\beta(i, j)=\beta(i, j \mid A, B, C, K)=\sum \frac{K A^{i+j+1}}{B^{i} C^{j}}$,

3) $\lambda(i, j)=\lambda(i, j \mid A, B, C, K)=\sum \frac{K^{2} B^{i+1} C^{j+1}}{A^{i+j}}$,

4) $\gamma(i, j)=\gamma(i, j \mid A, B, C, K)=\sum \frac{K A^{i+j-1}}{B^{i} C^{j}}$,

5) $\mu(i, j)=\mu(i, j \mid A, B, C, K)=\sum \frac{K^{2} B^{i-1} C^{j-1}}{A^{i+j}}$,

Proposition 5. With the above notations,

1) $\left\{\frac{A^{i+j}}{B^{i} C^{j}}, \frac{B^{i+j}}{C^{i} A^{j}}, \frac{C^{i+j}}{A^{i} B^{j}}\right\}$ are the roots of a monic equation

$$
t^{3}-\alpha(i, j) t^{2}+\alpha(-i,-j) t-1=0 .
$$

2) $\left\{\frac{K A^{i+j+1}}{B^{i} C^{j}}, \frac{K B^{i+j+1}}{C^{i} A^{j}}, \frac{K C^{i+j+1}}{A^{i} B^{j}}\right\}$ are the roots of a monic equation

$$
t^{3}-\beta(i, j) t^{2}+\lambda(i, j) t-K^{3} P=0 .
$$

3) $\left\{\frac{K A^{i+j-1}}{B^{i} C^{j}}, \frac{K B^{i+j-1}}{C^{i} A^{j}}, \frac{K C^{i+j-1}}{A^{i} B^{j}}\right\}$ are the roots of a monic equation

$$
t^{3}-\gamma(i, j) t^{2}+\mu(i, j) t-\frac{K^{3}}{P}=0 .
$$

Proof. For (1),

$$
\sum \frac{A^{i+j}}{B^{i} C^{j}}=\alpha(i, j)
$$




$$
\begin{gathered}
\sum\left(\frac{A^{i+j}}{B^{i} C^{j}} \cdot \frac{B^{i+j}}{C^{i} A^{j}}\right)=\sum \frac{A^{i} B^{j}}{C^{i+j}}=\sum \frac{B^{i} C^{j}}{A^{i+j}}=\alpha(-i,-j) . \\
\frac{A^{i+j}}{B^{i} C^{j}} \cdot \frac{B^{i+j}}{C^{i} A^{j}} \cdot \frac{C^{i+j}}{A^{i} B^{j}}=1 .
\end{gathered}
$$

Now statement (1) is clear. For (2),

$$
\begin{gathered}
\sum \frac{K A^{i+j+1}}{B^{i} C^{j}}=\beta(i, j) . \\
\sum\left(\frac{K A^{i+j+1}}{B^{i} C^{j}} \cdot \frac{K B^{i+j+1}}{C^{i} A^{j}}\right)=\sum \frac{K^{2} A^{i+1} B^{j+1}}{C^{i+j}}=\sum \frac{K^{2} B^{i+1} C^{j+1}}{A^{i+j}}=\lambda(i, j), \\
\frac{K A^{i+j+1}}{B^{i} C^{j}} \cdot \frac{K B^{i+j+1}}{C^{i} A^{j}} \cdot \frac{K C^{i+j+1}}{A^{i} B^{j}}=K^{3} A B C=K^{3} P .
\end{gathered}
$$

Now statement (2) is clear. The proof for (3) is similar and omitted.

Proposition 6. With the above notations,

1) $\alpha(i, j)=\alpha(i-1, j) Q-\alpha(i-2, j+1)-\alpha(i-1, j-1)$,
2) $\alpha(i, j)=\alpha(i, j-1) R-\alpha(i-1, j-1)-\alpha(i+1, j-2)$,
3) $\alpha(i, j)=\alpha(i+1, j) R-\alpha(i+2, j-1)-\alpha(i+1, j+1)$,
4) $\alpha(i, j)=\alpha(i, j+1) Q-\alpha(i+1, j+1)-\alpha(i-1, j+2)$,
5) $\beta(i, j)=\beta(i-1, j) Q-\beta(i-2, j+1)-\beta(i-1, j-1)$,
6) $\beta(i, j)=\beta(i, j-1) R-\beta(i-1, j-1)-\beta(i+1, j-2)$,
7) $\lambda(i, j)=\lambda(i-1, j) R-\lambda(i-2, j+1)-\lambda(i-1, j-1)$,
8) $\lambda(i, j)=\lambda(i, j-1) Q-\lambda(i-1, j-1)-\lambda(i+1, j-2)$,
9) $\gamma(i, j)=\gamma(i-1, j) Q-\gamma(i-2, j+1)-\gamma(i-1, j-1)$,
10) $\gamma(i, j)=\gamma(i, j-1) R-\gamma(i-1, j-1)-\gamma(i+1, j-2)$,
11) $\mu(i, j)=\mu(i-1, j) R-\mu(i-2, j+1)-\mu(i-1, j-1)$,
12) $\mu(i, j)=\mu(i, j-1) Q-\mu(i-1, j-1)-\mu(i+1, j-2)$.

Proof. For (1) we compute

$$
\begin{aligned}
\alpha(i, j) & =\sum\left(\frac{A^{i+j-1}}{B^{i-1} C^{j}} \cdot \frac{A}{B}\right) \\
& =\left(\sum \frac{A^{i+j-1}}{B^{i-1} C^{j}}\right)\left(\sum \frac{A}{B}\right)-\sum\left(\frac{A^{i+j-1}}{B^{i-1} C^{j}} \cdot \frac{B}{C}\right)-\sum\left(\frac{A^{i+j-1}}{B^{i-1} C^{j}} \cdot \frac{C}{A}\right) \\
& =\left(\sum \frac{A^{i+j-1}}{B^{i-1} C^{j}}\right)\left(\sum \frac{A}{B}\right)-\sum \frac{A^{i+j-1}}{B^{i-2} C^{j+1}}-\sum \frac{A^{i+j-2}}{B^{i-1} C^{j-1}} \\
& =\alpha(i-1, j) Q-\alpha(i-2, j+1)-\alpha(i-1, j-1) .
\end{aligned}
$$

For (2) we compute

$$
\begin{aligned}
\alpha(i, j) & =\sum\left(\frac{A^{i+j-1}}{B^{i} C^{j-1}} \cdot \frac{A}{C}\right) \\
& =\left(\sum \frac{A^{i+j-1}}{B^{i} C^{j-1}}\right)\left(\sum \frac{A}{C}\right)-\sum\left(\frac{A^{i+j-1}}{B^{i} C^{j-1}} \cdot \frac{B}{A}\right)-\sum\left(\frac{A^{i+j-1}}{B^{i} C^{j-1}} \cdot \frac{C}{B}\right) \\
& =\left(\sum \frac{A^{i+j-1}}{B^{i} C^{j-1}}\right)\left(\sum \frac{A}{C}\right)-\sum \frac{A^{i+j-2}}{B^{i-1} C^{j-1}}-\sum \frac{A^{i+j-1}}{B^{i+1} C^{j-2}} \\
& =\alpha(i, j-1) R-\alpha(i-1, j-1)-\alpha(i+1, j-2) .
\end{aligned}
$$


For (3) we compute

$$
\begin{aligned}
& \alpha(i, j) \\
& =\sum\left(\frac{A^{i+j+1}}{B^{i+1} C^{j}} \cdot \frac{B}{A}\right) \\
& =\left(\sum \frac{A^{i+j+1}}{B^{i+1} C^{j}}\right)\left(\sum \frac{B}{A}\right)-\sum\left(\frac{A^{i+j+1}}{B^{i+1} C^{j}} \cdot \frac{C}{B}\right)-\sum\left(\frac{A^{i+j+1}}{B^{i+1} C^{j}} \cdot \frac{A}{C}\right) \\
& =\left(\sum \frac{A^{i+j+1}}{B^{i+1} C^{j}}\right)\left(\sum \frac{B}{A}\right)-\sum \frac{A^{i+j+1}}{B^{i+2} C^{j-1}}-\sum \frac{A^{i+j+2}}{B^{i+1} C^{j+1}} \\
& =\alpha(i+1, j) R-\alpha(i+2, j-1)-\alpha(i+1, j+1) .
\end{aligned}
$$

For (4) we compute

$$
\begin{aligned}
& \alpha(i, j) \\
& =\sum \frac{A^{i+j+1}}{B^{i} C^{j+1}} \cdot \frac{C}{A} \\
& =\left(\sum \frac{A^{i+j+1}}{B^{i} C^{j+1}}\right)\left(\sum \frac{C}{A}\right)-\sum\left(\frac{A^{i+j+1}}{B^{i} C^{j+1}} \cdot \frac{A}{B}\right)-\sum\left(\frac{A^{i+j+1}}{B^{i} C^{j+1}} \cdot \frac{B}{C}\right) \\
& =\left(\sum \frac{A^{i+j+1}}{B^{i} C^{j+1}}\right)\left(\sum \frac{C}{A}\right)-\sum \frac{A^{i+j+2}}{B^{i+1} C^{j+1}}-\sum \frac{A^{i+j+1}}{B^{i-1} C^{j+2}} \\
& =\alpha(i, j+1) Q-\alpha(i+1, j+1)-\alpha(i-1, j+2) .
\end{aligned}
$$

For (5) we compute

$$
\begin{aligned}
& \beta(i, j) \\
& =\sum\left(\frac{K A^{i+j}}{B^{i-1} C^{j}} \cdot \frac{A}{B}\right) \\
& =\left(\sum \frac{K A^{i+j}}{B^{i-1} C^{j}}\right)\left(\sum \frac{A}{B}\right)-\sum\left(\frac{K A^{i+j}}{B^{i-1} C^{j}} \cdot \frac{B}{C}\right)-\sum\left(\frac{K A^{i+j}}{B^{i-1} C^{j}} \cdot \frac{C}{A}\right) \\
& =\left(\sum \frac{K A^{i+j}}{B^{i-1} C^{j}}\right)\left(\sum \frac{A}{B}\right)-\sum \frac{K A^{i+j}}{B^{i-2} C^{j+1}}-\sum \frac{K A^{i+j-1}}{B^{i-1} C^{j-1}} \\
& =\beta(i-1, j) Q-\beta(i-2, j+1)-\beta(i-1, j-1) .
\end{aligned}
$$

For (6) we compute

$$
\begin{aligned}
& \beta(i, j) \\
& =\sum\left(\frac{K A^{i+j}}{B^{i} C^{j-1}} \cdot \frac{A}{C}\right) \\
& =\left(\sum \frac{K A^{i+j}}{B^{i} C^{j-1}}\right)\left(\sum \frac{A}{C}\right)-\sum\left(\frac{K A^{i+j}}{B^{i} C^{j-1}} \cdot \frac{B}{A}\right)-\sum\left(\frac{K A^{i+j}}{B^{i} C^{j-1}} \cdot \frac{C}{B}\right) \\
& =\left(\sum \frac{K A^{i+j}}{B^{i} C^{j-1}}\right)\left(\sum \frac{A}{C}\right)-\sum \frac{K A^{i+j-1}}{B^{i-1} C^{j-1}}-\sum \frac{K A^{i+j}}{B^{i+1} C^{j-2}} \\
& =\beta(i, j-1) R-\beta(i-1, j-1)-\beta(i+1, j-2) .
\end{aligned}
$$


Similarly we can prove the rest of equations.

Proposition 7. With the above notations,

1) If $\alpha(1,1)$ is an integer and $\alpha(n, 0)$ are integers for all integer $n \geq 0$. Then for integers $i, j \geq 0$,

$$
\left\{\left(\frac{A^{i+j}}{B^{i} C^{j}}\right)^{n}+\left(\frac{B^{i+j}}{C^{i} A^{j}}\right)^{n}+\left(\frac{C^{i+j}}{A^{i} B^{j}}\right)^{n} \mid n=0,1,2, \cdots\right\}
$$

is an integer sequence.

2) If $\sum \alpha(-1,-1)$ is an integer and $\alpha(0, n)$ are integers for all integer $n \geq 0$. Then for integers $i \geq 0, j \geq 0$,

$$
\left\{\left(\frac{B^{i} C^{j}}{A^{i+j}}\right)^{n}+\left(\frac{C^{i} A^{j}}{B^{i+j}}\right)^{n}+\left(\frac{A^{i} B^{j}}{C^{i+j}}\right)^{n} \mid n=0,1,2, \cdots\right\}
$$

is an integer sequence.

3) If $\beta(1,1)$ and $\lambda(1,1)$ are integers and $\beta(n, 0)$ and $\lambda(n, 0)$ are integers for all integer $n \geq 0$. Then for integers $i \geq 0, j \geq 0$,

$$
\left\{\left(\frac{K A^{i+j+1}}{B^{i} C^{j}}\right)^{n}+\left(\frac{K B^{i+j+1}}{C^{i} A^{j}}\right)^{n}+\left(\frac{K C^{i+j+1}}{A^{i} B^{j}}\right)^{n} \mid n=0,1,2, \cdots\right\}
$$

is an integer sequence.

4) If $\gamma(1,1)$ and $\mu(1,1)$ are integers and $\gamma(n, 0)$ and $\mu(n, 0)$ are integers for all integer $n \geq 0$. Then for integers $i \geq 0, j \geq 0$,

$$
\left\{\left(\frac{K A^{i+j-1}}{B^{i} C^{j}}\right)^{n}+\left(\frac{K B^{i+j-1}}{C^{i} A^{j}}\right)^{n}+\left(\frac{K C^{i+j-1}}{A^{i} B^{j}}\right)^{n} \mid n=0,1,2, \cdots\right\}
$$

is an integer sequence.

Proof. We prove the first statement by induction on the sum $(i+j)$ that $\alpha(i, j)$ are integers for all $i, j \geq 0$.

Assume that all $\alpha(p, q)$ are integers for all $p, q \geq 0$ such that $(p+q)<(i+j)$.

Case 1. $i \geq 2, j \geq 1$. By Equation (1),

$$
\alpha(i, j)=\alpha(i-1, j) Q-\alpha(i-2, j+1)-\alpha(i-1, j-1)
$$

where

$$
\alpha(i-1, j), \alpha(i-2, j+1), \alpha(i-1, j-1)
$$

are integers by induction hypothesis.

Case 2. $i \geq 1, j \geq 2$. By Equation (2),

$$
\alpha(i, j)=\alpha(i, j-1) R-\alpha(i-1, j-1)-\alpha(i+1, j-2)
$$

where

$$
\alpha(i, j-1), \alpha(i-1, j-1), \alpha(i+1, j-2)
$$

are integers by induction hypothesis.

Case 3. $j=0$. Then $\alpha(i, j)$ is an integer by assumption. 
Case 4. $i=0$. Then $\alpha(i, j)$ is an integer by assumption.

It follows that $\alpha(i, j)$ is an integer in all cases.

By induction, $\alpha(i, j)$ is an integer for all $0 \leq i, j \leq 1$.

It follows from Corollary 2 that

$$
\left\{\left(\frac{A^{i+j}}{B^{i} C^{j}}\right)^{n}+\left(\frac{B^{i+j}}{C^{i} A^{j}}\right)^{n}+\left(\frac{C^{i+j}}{A^{i} B^{j}}\right)^{n} \mid n=0, \pm 1, \pm 2, \cdots\right\}
$$

is an integer sequence.

We can prove the other statements in a similar fashion.

Remark 8. With above notations,

1) we will call this sequence

$$
\left\{\left(\frac{A^{i+j}}{B^{i} C^{j}}\right)^{n}+\left(\frac{B^{i+j}}{C^{i} A^{j}}\right)^{n}+\left(\frac{C^{i+j}}{A^{i} B^{j}}\right)^{n} \mid n=0,1,2, \cdots\right\}
$$

an $\alpha$ sequence.

2) we will call this sequence

$$
\left\{\left(\frac{K A^{i+j+1}}{B^{i} C^{j}}\right)^{n}+\left(\frac{K B^{i+j+1}}{C^{i} A^{j}}\right)^{n}+\left(\frac{K C^{i+j+1}}{A^{i} B^{j}}\right)^{n} \mid n=0,1,2, \cdots\right\}
$$

a $\beta$ sequence.

3) we will call this sequence

$$
\left\{\left(\frac{K A^{i+j-1}}{B^{i} C^{j}}\right)^{n}+\left(\frac{K B^{i+j-1}}{C^{i} A^{j}}\right)^{n}+\left(\frac{K C^{i+j-1}}{A^{i} B^{j}}\right)^{n} \mid n=0,1,2, \cdots\right\}
$$

a $\gamma$ sequence.

In later sections, we will need to use the following formulas.

Proposition 9. With the above notations,

$$
\begin{gathered}
\sum \frac{A}{B}+\sum \frac{A}{C}=\left(\sum A\right)\left(\sum \frac{1}{A}\right)-3 \\
\left(\sum \frac{A}{B}\right)\left(\sum \frac{A}{C}\right)=\left(\frac{1}{P}\right)\left(\sum A^{3}\right)+P\left(\sum \frac{1}{A^{3}}\right)+3 .
\end{gathered}
$$

For integers $i, j$,

$$
\begin{gathered}
\sum A^{i} B^{j}+\sum A^{j} B^{i}=\left(\sum A^{i}\right)\left(\sum A^{j}\right)-\left(\sum A^{i+j}\right) \\
\left(\sum A^{i} B^{j}\right)\left(\sum A^{j} B^{i}\right)=(\Pi A)^{i+j}\left(\sum A^{-i-j}\right)+(\Pi A)^{i} \sum A^{2 j-i}+(\Pi A)^{j} \sum A^{2 i-j} .
\end{gathered}
$$

Proof. This can be verified by simple algebra.

\section{Power Sums of Sine Values}

In this section we will compute the sum of powers and mixed powers of sine values. In this section and Section $4,5,6$, let

$$
A=\sin 2 \theta, B=\sin 4 \theta, C=\sin 8 \theta,
$$


The following result can be easily proved using equations in [4] [5].

Proposition 10. With above notations, $\{A, B, C\}$ are the roots of the equation

$$
x^{3}-\frac{\sqrt{7}}{2} x^{2}+\frac{\sqrt{7}}{8}=0
$$

and $\left\{4 A^{2}, 4 B^{2}, 4 C^{2}\right\}$ are the roots of the equation

$$
x^{3}-7 x^{2}+14 x-7=0 .
$$

Definition 11. For an integer $n$, let

$$
S(n)=A^{n}+B^{n}+C^{n} .
$$

For integers $i, j$, let

$$
U(i, j)=\sum A^{i} B^{j}
$$

Let

$$
D=A B C=-\frac{\sqrt{7}}{8} .
$$

Proposition 12. With the above notations, $S(n)$ satisfy the following recurrence relations:

$$
\begin{gathered}
S(n)=\frac{\sqrt{7}}{2} S(n-1)-\frac{\sqrt{7}}{8} S(n-3), \\
S(-n)=4 S(-n+2)-\frac{8 \sqrt{7}}{7} S(-n+3) .
\end{gathered}
$$

Proof. The recurrence relations follow easily from the equations in Proposition 10.

Example 13. With the above notations, for $n=0, \cdots, 10$

$$
\begin{gathered}
S(n)=3, \frac{\sqrt{7}}{2}, \frac{7}{4}, \frac{\sqrt{7}}{2}, \frac{21}{16}, \frac{7 \sqrt{7}}{16}, \frac{35}{32}, \frac{49 \sqrt{7}}{128}, \frac{245}{256}, \frac{175 \sqrt{7}}{512}, \frac{441}{512}, \cdots \\
S(-n)=3,0,8,-\frac{24 \sqrt{7}}{7}, 32,-\frac{160 \sqrt{7}}{7}, \frac{1088}{7},-128 \sqrt{7}, \frac{5632}{7}, \\
- \\
-\frac{33792 \sqrt{7}}{49}, \frac{29296}{7},-\frac{180224 \sqrt{7}}{49}, \cdots
\end{gathered}
$$

Proposition 14. With the above notations, $U(i, j)$ satisfy the following recurrence relations.

$$
\begin{aligned}
& U(i, j)=\frac{\sqrt{7}}{2} U(i-1, j)-\frac{\sqrt{7}}{8} U(i-3, j), \\
& U(i, j)=\frac{\sqrt{7}}{2} U(i, j-1)-\frac{\sqrt{7}}{8} U(i, j-3) .
\end{aligned}
$$

Proof. The recurrence relations follow easily from the equations in Proposition 10.

Lemma 15. With the above notations, list of $U(i, j), i, j=1, \cdots, 10$ : 


$$
\begin{aligned}
& 0, \frac{\sqrt{7}}{4}, \frac{7}{16}, \frac{7 \sqrt{7}}{32}, \frac{35}{64}, \frac{7 \sqrt{7}}{32}, \frac{147}{256}, \frac{7 \sqrt{7}}{32}, \frac{147}{256}, \frac{441 \sqrt{7}}{2048}, \\
& \frac{\sqrt{7}}{8}, \frac{7}{8}, \frac{7 \sqrt{7}}{32}, \frac{21}{32}, \frac{7 \sqrt{7}}{32}, \frac{147}{256}, \frac{105 \sqrt{7}}{512}, \frac{539}{1024}, \frac{49 \sqrt{7}}{256}, \frac{251}{512}, \\
& 0, \quad \frac{7 \sqrt{7}}{32}, \frac{21}{64}, \frac{21 \sqrt{7}}{128}, \frac{49}{128}, \frac{77 \sqrt{7}}{512}, \frac{49}{128}, \frac{147 \sqrt{7}}{1024}, \frac{95}{256}, \frac{141 \sqrt{7}}{1024}, \\
& 0, \quad \frac{35}{64}, \frac{7 \sqrt{7}}{64}, \frac{49}{128}, \frac{63 \sqrt{7}}{512}, \frac{343}{1024}, \frac{245 \sqrt{7}}{2048}, \frac{637}{2048}, \frac{931 \sqrt{7}}{8192}, \frac{75}{256}, \\
& \frac{-7}{64}, \frac{21 \sqrt{7}}{128}, \frac{49}{256}, \frac{7 \sqrt{7}}{64}, \frac{245}{1024}, \frac{49 \sqrt{7}}{512}, \frac{245}{1024}, \frac{735 \sqrt{7}}{8192}, \frac{59}{256}, \frac{349 \sqrt{7}}{4096}, \\
& \frac{-7 \sqrt{7}}{128}, \frac{49}{128}, \frac{7 \sqrt{7}}{128}, \frac{245}{1024}, \frac{147 \sqrt{7}}{2048}, \frac{833}{4096}, \frac{147 \sqrt{7}}{2048}, \frac{193}{1024}, \frac{141 \sqrt{7}}{2048}, \frac{729}{4096}, \\
& \frac{-49}{256}, \frac{63 \sqrt{7}}{512}, \frac{49}{512}, \frac{147 \sqrt{7}}{2048}, \frac{147}{1024}, \frac{245 \sqrt{7}}{4096}, \frac{75}{512}, \frac{453 \sqrt{7}}{8192}, \frac{579}{4096}, \frac{429 \sqrt{7}}{8192}, \\
& \frac{-21 \sqrt{7}}{256}, \frac{147}{512}, \frac{49 \sqrt{7}}{2048}, \frac{637}{4096}, \frac{343 \sqrt{7}}{8192}, \frac{257}{2048}, \frac{355 \sqrt{7}}{8192}, \frac{59}{512}, \frac{343 \sqrt{7}}{8192}, \frac{445}{4096}, \\
& \frac{-245}{1024}, \frac{49 \sqrt{7}}{512}, \frac{147}{4096}, \frac{49 \sqrt{7}}{1024}, \frac{343}{4096}, \frac{153 \sqrt{7}}{4096}, \frac{729}{8192}, \frac{557 \sqrt{7}}{16384}, \frac{177}{2048}, \frac{525 \sqrt{7}}{16384}, \\
& \frac{-49 \sqrt{7}}{512}, \frac{931}{4096}, \frac{49 \sqrt{7}}{8192}, \frac{429}{4096}, \frac{49 \sqrt{7}}{2048}, \frac{643}{8192}, \frac{429 \sqrt{7}}{16384}, \frac{579}{8192}, \frac{209 \sqrt{7}}{8192}, \frac{17}{256},
\end{aligned}
$$

Proof. All the computations are similar. We will only prove $U(3,5)$ and $U(5,3)$ By Proposition 29,

$$
\begin{gathered}
U(5,3)+U(3,5)=S(3) S(5)-S(8)=\frac{147}{256} . \\
U(5,3) U(3,5)=D^{8} S(-8)+D^{5} S(1)+D^{3} S(7)=\frac{2401}{32768} .
\end{gathered}
$$

Next we solve the equation

$$
t^{2}-\frac{147}{256} t+\frac{2401}{32768}=0
$$

to get roots $\left\{\frac{49}{256}, \frac{49}{1286}\right\}$.

Note that $A \approx 0.782, B \approx 0.975, C \approx-0.434$.

Then

$$
\begin{aligned}
& U(5,3)=A^{5} B^{3}+B^{5} C^{3}+C^{5} A^{3} \approx 0.191 \\
& U(3,5)=A^{3} B^{5}+B^{3} C^{5}+C^{3} A^{5} \approx 0.382 .
\end{aligned}
$$

It follows that

$$
U(5,3)=\frac{49}{256}, U(3,5)=\frac{49}{128}
$$

Remark 16. Alternatively, we can first use above methods to compute $U(i, j)$ for $i, j=0,1,2,3$, then compute the other cases using recurrence formula in Proposition 14. 


\section{Alpha Sequences of Sine Values}

In this section we will study the $\alpha$ sequences for sine values.

Recall that

$$
Q=\sum \frac{A}{B}, R=\sum \frac{A}{C}
$$

and for integers $i, j$,

$$
\alpha(i, j)=\sum \frac{A^{i+j}}{B^{i} C^{j}} .
$$

Lemma 17.

$$
Q=-2, R=-1 \text {. }
$$

Proof. By Proposition 9

$$
\begin{gathered}
Q+R=S(1) S(-1)-3=\left(\frac{\sqrt{7}}{2}\right)(0)-3=-3 . \\
Q R=\frac{S(3)}{D}+D S(-3)+3=\left(\frac{\sqrt{7}}{2}\right)\left(\frac{-8}{\sqrt{7}}\right)+\left(\frac{-\sqrt{7}}{8}\right)\left(\frac{-24 \sqrt{7}}{7}\right)+3=2 .
\end{gathered}
$$

Then $\{Q, R\}=\{-2,-1\}$ are the roots of the equation:

$$
t^{2}+3 t+2=0 .
$$

Note that $A \approx 0.782, B \approx 0.975, C \approx-0.434$. Then $Q \approx-2.0, R \approx-1.0$ It follows that $Q=-2, R=-1$.

Proposition 18. With the above notations, $\left\{\frac{A}{B}, \frac{B}{C}, \frac{C}{A}\right\}$ are roots of equation

$$
t^{3}+2 t^{2}-t-1=0
$$

Proof. By Lemma 17,

$$
\begin{gathered}
\frac{A}{B}+\frac{B}{C}+\frac{C}{A}=-2, \\
\frac{A}{B} \cdot \frac{B}{C}+\frac{B}{C} \cdot \frac{C}{A}+\frac{C}{A} \cdot \frac{A}{B}=\frac{A}{C}+\frac{B}{A}+\frac{C}{B}=-1, \\
\frac{A}{B} \cdot \frac{B}{C} \cdot \frac{C}{A}=1,
\end{gathered}
$$

It follows that $\frac{A}{B}, \frac{B}{C}, \frac{C}{A}$ are the roots of the above equation.

\section{Proposition 19.}

$$
\alpha(0,0)=3, \alpha(1,0)=-2, \alpha(2,0)=6,
$$

and for $n \geq 0, \alpha(n, 0)$ satisfies recurrence equation:

$$
\begin{gathered}
\alpha(n, 0)=-2 \alpha(n-1,0)+\alpha(n-2,0)+\alpha(n-3,0), \\
\alpha(0,0)=3, \alpha(0,1)=-1, \alpha(0,2)=5,
\end{gathered}
$$

and for $n \geq 0, \alpha(0, n)$ satisfies recurrence equation: 


$$
\alpha(0, n)=-\alpha(0, n-1)+2 \alpha(0, n-2)-\alpha(0, n-3) .
$$

Proof. Recall that

$$
\alpha(i, j)=\sum \frac{A^{i+j}}{B^{i} C^{j}} .
$$

Then all equations follow easily from Proposition 18.

Theorem 20.

$$
\left\{\left(\frac{\sin 2 \theta}{\sin 4 \theta}\right)^{n}+\left(\frac{\sin 4 \theta}{\sin 8 \theta}\right)^{n}+\left(\frac{\sin 8 \theta}{\sin 2 \theta}\right)^{n} \mid n=0, \pm 1, \pm 2, \cdots\right\}
$$

is a two-sided integer sequence.

Proof. This follows easily from Proposition 19 and Proposition 7.

Example 21. For $n=0,1,2,3, \cdots$

$$
\begin{gathered}
\{\alpha(n, 0) \mid n=0,1,2,3, \cdots\}=\{3,-2,6,-11,26,-57,129,-289, \cdots\} \quad[\text { Sloane } \underline{\text { A033304 }}] \\
\{\alpha(0, n) \mid n=0,1,2,3, \cdots\}=\{3,-1,5,-4,13,-16,38,-57, \cdots\} \quad[\text { Barry } \underline{\text { A094648 }]}
\end{gathered}
$$

Lemma 22.

$$
\alpha(1,1)=-4, \alpha(-1,-1)=3
$$

Proof.

$$
\begin{gathered}
\alpha(1,1)=\sum \frac{A^{2}}{B C}=\frac{1}{D} \sum A^{3}=\frac{S(3)}{D}=-4, \\
\alpha(-1,-1)=\sum \frac{B C}{A^{2}}=D \sum \frac{1}{A^{3}}=D S(-3)=3 .
\end{gathered}
$$

Theorem 23. For integers $i, j$,

$$
\left\{\left(\frac{\sin ^{i+j} 2 \theta}{\sin ^{i} 4 \theta \sin ^{j} 8 \theta}\right)^{n}+\left(\frac{\sin ^{i+j} 4 \theta}{\sin ^{i} 8 \theta^{i} \sin ^{j} 2 \theta}\right)^{n}+\left(\frac{\sin ^{i+j} 8 \theta}{\sin ^{i} 2 \theta^{i} \sin ^{j} 4 \theta}\right)^{n} \mid n=0, \pm 1, \pm 2, \cdots\right\}
$$

is a two-sided integer sequence.

Proof. By Lemma 22 and Corollary 2, Proposition 7, Proposition 21.

Remark 24. Using our approach, we can generate two-sided integer sequences as follows.

1) For each $(i, j), i, j \geq 0$, we can compute $\alpha(i, j), \alpha(-i,-j)$ using recurrence relations in Proposition 6.

2) Let

$$
X=\frac{A^{i+j}}{B^{i} C^{j}}, Y=\frac{B^{i+j}}{C^{i} A^{j}}, Z=\frac{C^{i+j}}{A^{i} B^{j}} .
$$

Then $\{X, Y, Z\}$ are the roots for equation:

$$
t^{3}-\alpha(i, j) t^{2}+\alpha(-i,-j) t-1=0 .
$$

3) Let $p(n)=\sum X^{n}$. Then

$$
p(0)=3, p(1)=\alpha(i, j), p(2)=\alpha(i, j)^{2}-2 \alpha(-i,-j) .
$$


4) For $n \geq 3$ and $n<0$ use the recurrence relation

$$
p(n)=\alpha(i, j) p(n-1)-\alpha(-i,-j) p(n-2)+p(n-3)
$$

to get $p(n)$.

\section{Remark 25. Note that}

1 ) integer sequence for $(n, 0)$ is a subsequence for the integer sequence for $(1,0)$.

2) integer sequence for $(0, n)$ is a subsequence for the integer sequence for $(0,1)$.

Example 26. For integers $i, j$ let

$$
E Q(i, j)=t^{3}-\alpha(i, j) t^{2}+\alpha(-i,-j) t-1=0,
$$

and let $S E Q 1, S E Q 2$ be the associated sequences. Here is a list of integer equations and the associated integer sequences.

1) $E Q(0,0)=t^{3}-3 t^{2}+3 t-1=0$.

$S E Q 1=3,3,3,3,3,3,3, \cdots$

$S E Q 2=3,3,3,3,3,3,3, \cdots$

2) $E Q(0,1)=t^{3}+t^{2}-2 t-1=0$.

$S E Q 1=3,-1,5,-4,13,-16,38, \cdots \quad$ [Barry A094648]

$S E Q 2=3,-2,6,-11,26,-57,129, \cdots \quad$ [Sloane A033304]

3) $E Q(1,0)=t^{3}+2 t^{2}-t-1=0$.

$S E Q 1=3,-2,6,-11,26,-57,129, \cdots$

$S E Q 2=3,-1,5,-4,13,-16,38, \cdots$

4) $E Q(0,2)=t^{3}-5 t^{2}+6 t-1=0$.

$S E Q 1=3,5,13,38,117,370,1186, \cdots$

$S E Q 2=3,6,26,129,650,3281,16565, \cdots$

5) $E Q(1,1)=t^{3}+4 t^{2}+3 t-1=0$.

$S E Q 1=3,-4,10,-25,66,-179,493, \cdots \quad$ [Wang A274220]

$S E Q 2=3,3,17,66,269,1088,4406, \cdots \quad$ [Witula A215076]

6) $E Q(2,0)=t^{3}-6 t^{2}+5 t-1=0$.

$S E Q 1=3,6,26,129,650,3281,16565, \cdots$

$S E Q 2=3,5,13,38,117,370,1186, \cdots$

7) $E Q(0,3)=t^{3}+4 t^{2}-11 t-1=0$.

$S E Q 1=3,-4,38,-193,1186,-6829,40169, \cdots$

$S E Q 2=3,-11,129,-1460,16565,-187926,2131986, \cdots$

8) $E Q(1,2)=t^{3}+t^{2}-9 t-1=0$.

SEQ1 $=3,-1,19,-25,195,-401,2131, \cdots \quad$ [Wang A274075]

$S E Q 2=3,-9,83,-753,6851,-62329,567059, \cdots \quad$ [Wang A274032]

9) $E Q(2,1)=t^{3}-5 t^{2}-8 t-1=0$.

$S E Q 1=3,5,41,248,1573,9890,62282, \cdots$

$S E Q 2=3,-8,54,-389,2834,-20673,150825, \cdots$

10) $E Q(3,0)=t^{3}+11 t^{2}-4 t-1=0$.

$S E Q 1=3,-11,129,-1460,16565,-187926,2131986, \cdots$

$S E Q 2=3,-4,38,-193,1186,-6829,40169, \cdots$ 
11) $E Q(0,4)=t^{3}-13 t^{2}+26 t-1=0$.

$S E Q 1=3,13,117,1186,12389,130338,1373466, \cdots$

$S E Q 2=3,26,650,16565,422266,10764221,274396853, \cdots$

12) $E Q(1,3)=t^{3}+9 t^{2}+20 t-1=0$.

$S E Q 1=3,-9,41,-186,845,-3844,17510, \cdots \quad$ [Wang A320918]

$S E Q 2=3,20,418,8543,174642,3570145,72983221, \cdots$

13) $E Q(2,2)=t^{3}-10 t^{2}+17 t-1=0$.

$S E Q 1=3,10,66,493,3818,29865,234237, \cdots$

$S E Q 2=3,17,269,4406,72229,1184102,19411850, \cdots$

14) $E Q(3,1)=t^{3}+15 t^{2}+12 t-1=0$.

$S E Q 1=3,-15,201,-2832,40053,-566610,8015682, \cdots$

$S E Q 2=3,12,174,2271,29874,392727,5163105, \cdots$

15) $E Q(4,0)=t^{3}-26 t^{2}+13 t-1=0$.

$S E Q 1=3,26,650,16565,422266,10764221,274396853, \cdots$

$S E Q 2=3,13,117,1186,12389,130338,1373466, \cdots$

16) $E Q(0,5)=t^{3}+16 t^{2}-57 t-1=0$.

SEQ1 $=3,-16,370,-6829,130338,-2474291,47011093, \cdots$

$S E Q 2=3,-57,3281,-187926,10764221,-616564132,35316195134, \cdots$

17) $E Q(1,4)=t^{3}-3 t^{2}-46 t-1=0$.

$S E Q 1=3,3,101,444,5981,38468,390974, \cdots \quad$ [Witula A215560]

$S E Q 2=3,-46,2110,-96919,4451898,-204494441,9393291673, \cdots$

18) $E Q(2,3)=t^{3}-6 t^{2}-37 t-1=0$.

$S E Q 1=3,6,110,885,9386,89171,883193, \cdots$

$S E Q 2=3,-37,1357,-49984,1841229,-67824212,2498398486, \cdots$

19) $E Q(3,2)=t^{3}+16 t^{2}-29 t-1=0$.

SEQ1 $=3,-16,314,-5485,96850,-1708351,30136781, \cdots$

$S E Q 2=3,-29,873,-25778,761501,-22495104,664516254, \cdots$

20) $E Q(4,1)=t^{3}-31 t^{2}-25 t-1=0$.

$S E Q 1=3,31,1011,32119,1020995,32454831,1031656755, \cdots \quad$ [Wang,

A274592]

SEQ2 $=3,-25,563,-13297,314947,-7460905,176745971, \cdots \quad$ [Wang A248417]

21) $E Q(5,0)=t^{3}+57 t^{2}-16 t-1=0$.

$S E Q 1=3,-57,3281,-187926,10764221,-616564132,35316195134, \cdots$

$S E Q 2=3,-16,370,-6829,130338,-2474291,47011093, \cdots$

\section{Beta Sequences of Sine Values}

In this section we will study the $\beta$ sequences for sine values.

Let $K=2 \sqrt{7}$ in the formula for $\beta(i, j)$ and $\lambda(i, j)$. Then

$$
\beta(i, j)=\sum \frac{2 \sqrt{7} A^{i+j+1}}{B^{i} C^{j}}, \lambda(i, j)=\sum \frac{28 B^{i+1} C^{j+1}}{A^{i+j}} .
$$

Lemma 27. With the above notations,

1) $\beta(0,0)=7$, 

2) $\beta(1,0)=-7$,
3) $\beta(0,1)=0$,
4) $\beta(2,0)=28$,
5) $\beta(0,2)=21$,
6) $\beta(1,1)=-21$,
7) $\lambda(0,0)=0$,
8) $\lambda(1,0)=-49$,
9) $\lambda(0,1)=-49$,
10) $\lambda(2,0)=49$,
11) $\lambda(0,2)=98$,
12) $\lambda(1,1)=98$.

Proof. To prove (1),

$$
\beta(0,0)=2 \sqrt{7} \sum A=2 \sqrt{7} \cdot \frac{\sqrt{7}}{2}=7 .
$$

To prove (2),

$$
\beta(1,0)=2 \sqrt{7} \sum \frac{A^{2}}{B}=\frac{2 \sqrt{7}}{D} \sum A B^{3}=\frac{2 \sqrt{7}}{D} U(1,3)=-7 .
$$

To prove (3),

$$
\beta(0,1)=2 \sqrt{7} \sum \frac{A^{2}}{C}=\frac{2 \sqrt{7}}{D} \sum A^{3} B=\frac{2 \sqrt{7}}{D} U(3,1)=0 .
$$

To prove (4),

$$
\beta(2,0)=2 \sqrt{7} \sum \frac{A^{3}}{B^{2}}=\frac{2 \sqrt{7}}{D^{2}} \sum A^{2} B^{5}=\frac{2 \sqrt{7}}{D^{2}} U(2,5)=28 .
$$

To prove (5),

$$
\beta(0,2)=2 \sqrt{7} \sum \frac{A^{3}}{C^{2}}=\frac{2 \sqrt{7}}{P^{2}} \sum A^{5} B^{2}=\frac{2 \sqrt{7}}{D^{2}} U(5,2)=7 .
$$

To prove (6),

$$
\beta(1,1)=2 \sqrt{7} \sum \frac{A^{3}}{B C}=\frac{2 \sqrt{7}}{D} \sum A^{4}=\frac{2 \sqrt{7}}{D} S(4)=-21 .
$$

To prove (7),

$$
\lambda(0,0)=28 \sum B C=\frac{28}{D} \sum \frac{1}{A}=\frac{28}{D} S(-1)=0 .
$$

To prove (8),

$$
\lambda(1,0)=28 \sum \frac{B^{2} C}{A}=\frac{28}{D} \sum A^{3} B^{2}=\frac{28}{D} U(3,2)=-49 .
$$

To prove (9),

$$
\lambda(0,1)=28 \sum \frac{B C^{2}}{A}=\frac{28}{D} \sum A^{2} B^{3}=\frac{28}{D} U(2,3)=-49 .
$$

To prove (10), 


$$
\lambda(2,0)=28 \sum \frac{B^{3} C}{A^{2}}=\frac{28}{D^{2}} \sum A^{5} B^{3}=\frac{28}{D^{2}} U(5,3)=49 .
$$

To prove (11),

$$
\lambda(0,2)=28 \sum \frac{B C^{3}}{A^{2}}=\frac{28}{D^{2}} \sum A^{3} B^{5}=\frac{28}{D^{2}} U(3,5)=98 .
$$

To prove (12),

$$
\lambda(1,1)=28 \sum \frac{B^{2} C^{2}}{A^{2}}=28 D^{2} \sum \frac{1}{A^{4}}=28 D^{2} S(-4)=98 .
$$

Proposition 28. With the above notations,

1) $\beta(n, 0)=-2 \beta(n-1,0)+\beta(n-2,0)+\beta(n-3,0)$.

2) $\beta(0, n)=-\beta(0, n-1)+2 \beta(0, n-2)+\beta(0, n-3)$,

3) $\lambda(n, 0)=-\lambda(n-1,0)+2 \lambda(n-2,0)+\lambda(n-3,0)$,

4) $\lambda(0, n)=-2 \lambda(0, n-1)+\lambda(0, n-2)+\lambda(0, n-3)$.

Proof. By Proposition 18, $\left\{\frac{A}{B}, \frac{B}{C}, \frac{C}{A}\right\}$ are roots of equation

$$
t^{3}+2 t^{2}-t-1=0
$$

It follows that

$$
\begin{aligned}
& \left(\frac{A}{B}\right)^{n}=-2\left(\frac{A}{B}\right)^{n-1}+\left(\frac{A}{B}\right)^{n-2}+\left(\frac{A}{B}\right)^{n-3}, \\
& \left(\frac{B}{C}\right)^{n}=-2\left(\frac{B}{C}\right)^{n-1}+\left(\frac{B}{C}\right)^{n-2}+\left(\frac{B}{C}\right)^{n-3}, \\
& \left(\frac{C}{A}\right)^{n}=-2\left(\frac{C}{A}\right)^{n-1}+\left(\frac{C}{A}\right)^{n-2}+\left(\frac{C}{A}\right)^{n-3} .
\end{aligned}
$$

We now compute

$$
\begin{aligned}
\beta(n, 0) & =2 \sqrt{7} \sum \frac{A^{n+1}}{B^{n}}=2 \sqrt{7} \sum A\left(\frac{A}{B}\right)^{n} \\
& =2 \sqrt{7} \sum A\left(-2\left(\frac{A}{B}\right)^{n-1}+\left(\frac{A}{B}\right)^{n-2}+\left(\frac{A}{B}\right)^{n-3}\right) \\
& =-2 \cdot 2 \sqrt{7} \sum \frac{A^{n}}{B^{n-1}}+2 \sqrt{7} \sum \frac{A^{n-1}}{B^{n-2}}+2 \sqrt{7} \sum \frac{A^{n-2}}{B^{n-3}} \\
& =-2 \beta(n-1,0)+\beta(n-2,0)+\beta(n-3,0) .
\end{aligned}
$$

Similarly, we can prove other equations.

Proposition 29. With the above notations,

1) $\beta(n, 0)$ are integers for all integer $n \geq 0$,

2) $\beta(0, n)$ are integers for all integer $n \geq 0$,

3) $\lambda(n, 0)$ are integers for all integer $n \geq 0$,

4) $\lambda(0, n)$ are integers for all integer $n \geq 0)$

Proof. We will prove (1) by induction on $n \geq 0$.

By Lemma $27, \beta(0,0), \beta(1,0), \beta(2,0)$ are integers. Assume that $\beta(k, 0)$ are integers for all $k=0,1, \cdots,(n-1)$. By Proposition 27 


$$
\beta(n, 0)=-2 \beta(n-1,0)+\beta(n-2,0)+\beta(n-3,0) .
$$

This shows that $\beta(n, 0)$ is an integer. It follows by induction that $\beta(n, 0)$ is an integer for all $n \geq 0$. This proves (1).

Similarly We can prove other statements by induction on $n \geq 0$.

Theorem 30. For integers $i, j$,

$$
\left\{\left(\frac{2 \sqrt{7} \sin ^{i+j+1} 2 \theta}{\sin ^{i} 4 \theta \sin ^{j} 8 \theta}\right)^{n}+\left(\frac{2 \sqrt{7} \sin ^{i+j+1} 4 \theta}{\sin ^{i} 8 \theta \sin ^{j} 2 \theta}\right)^{n}+\left(\frac{2 \sqrt{7} \sin ^{i+j+1} 8 \theta}{\sin ^{i} 2 \theta \sin ^{j} 4 \theta}\right)^{n} \mid n=0,1,2, \cdots\right\}
$$

are integer sequences.

Proof. This follows easily from Proposition 7, Lemma 27 and Propositions 28, 26.

Remark 31. Note that

$$
\frac{2 \sqrt{7} A^{i+j+1}}{B^{i} C^{j}} \cdot \frac{2 \sqrt{7} B^{i+j+1}}{C^{i} A^{j}} \cdot \frac{2 \sqrt{7} C^{i+j+1}}{A^{i} B^{j}}=-49 .
$$

By Proposition 5

$$
\left\{\frac{2 \sqrt{7} A^{i+j+1}}{B^{i} C^{j}}, \frac{2 \sqrt{7} B^{i+j+1}}{C^{i} A^{j}}, \frac{2 \sqrt{7} C^{i+j+1}}{A^{i} B^{j}}\right\}
$$

are the roots of a monic equation

$$
E Q(i, j)=t^{3}-\beta(i, j) t^{2}+\lambda(i, j) t+49=0 .
$$

Let $\operatorname{SEQ}(i, j)$ be the associated sequence.

Example 32 With the above notations,

1) $E Q(0,0)=t^{3}-7 t^{2}+49=0$.

$S E Q=3,7,49,196,1029,4802,24010, \cdots \quad$ [Wang A275830]

2) $E Q(0,1)=t^{3}-49 t+49=0$.

$S E Q=3,0,98,-147,4802,-12005,242501, \cdots$

3) $E Q(1,0)=t^{3}+7 t^{2}-49 t+49=0$.

$S E Q=3,-7,147,-1519,18179,-208887,2427411, \cdots$

4) $E Q(0,2)=t^{3}-21 t^{2}+98 t+49=0$.

$S E Q=3,21,245,2940,36701,470596,6141758, \cdots$

5) $E Q(1,1)=t^{3}+21 t^{2}+98 t+49=0$.

$S E Q=3,-21,245,-3234,44933,-638666,9167018, \cdots$

6) $E Q(2,0)=t^{3}-28 t^{2}+49 t+49=0$.

$S E Q=3,28,686,17689,460306,11988193,312247649, \cdots$

7) $E Q(0,3)=t^{3}+14 t^{2}-245 t+49=0$.

$S E Q=3,-14,686,-13181,353290,-8209019,202128185, \cdots$

8) $E Q(1,2)=t^{3}+7 t^{2}-196 t+49=0$.

$S E Q=3,-7,441,-4606,119021,-1757532,35856534, \cdots$

9) $E Q(2,1)=t^{3}-28 t^{2}-147 t+49=0$.

$S E Q=3,28,1078,34153,1113378,36142253,1173976153, \cdots$

10) $E Q(3,0)=t^{3}+56 t^{2}-147 t+49=0$.

$S E Q=3,-56,3430,-200459,11732658,-686664391,40187729113, \cdots$ 
11) $E Q(0,4)=t^{3}-56 t^{2}+539 t+49=0$. $S E Q=3,56,2058,84917,3643346,158156271,6888826749, \cdots$

12) $E Q(1,3)=t^{3}+42 t^{2}+441 t+49=0$. $S E Q=3,-42,882,-18669,397194,-8492337,182430381, \cdots$

13) $E Q(2,2)=t^{3}-49 t^{2}+343 t+49=0$. $S E Q=3,49,1715,67081,2696323,109027009,4414197683, \cdots$

14) $E Q(3,1)=t^{3}+77 t^{2}+294 t+49=0$. $S E Q=3,-77,5341,-388766,28368501,-2070339082,151094819554, \cdots$

15) $E Q(4,0)=t^{3}-133 t^{2}+196 t+49=0$. $S E Q=3,133,17297,2274286,299083309,39331472488,5172354072326, \cdots$

\section{Gamma Sequences of Sine Values}

In this section we will study the $\gamma$ sequences for sine values. Let $K=\frac{\sqrt{7}}{2}$ in the formula for $\gamma(i, j)$ and $\mu(i, j)$. Then

$$
\gamma(i, j)=\sum \frac{\sqrt{7} A^{i+j-1}}{2 B^{i} C^{j}}, \mu(i, j)=\sum \frac{7 B^{i-1} C^{j-1}}{4 A^{i+j}} .
$$

Lemma 33. With the above notations,
1) $\gamma(0,0)=0$,
2) $\gamma(1,0)=0$
3) $\gamma(0,1)=0$,
4) $\gamma(2,0)=7$,
5) $\gamma(0,2)=7$,
6) $\gamma(1,1)=-7$,
7) $\mu(0,0)=-7$,
8) $\mu(1,0)=-7$,
9) $\mu(0,1)=-7$,
10) $\mu(2,0)=0$,
11) $\mu(0,2)=7$,
12) $\mu(1,1)=14$.

Proof. The proof is similar to the proof of Lemma 25.

Proposition 34. With the above notations,
1) $\gamma(n, 0)=-2 \gamma(n-1,0)+\gamma(n-2,0)+\gamma(n-3,0)$,
2) $\gamma(0, n)=-\gamma(0, n-1)+2 \gamma(0, n-2)+\gamma(0, n-3)$,
3) $\mu(n, 0)=-\mu(n-1,0)+2 \mu(n-2,0)+\mu(n-3,0)$,
4) $\mu(0, n)=-2 \mu(0, n-1)+\mu(0, n-2)+\mu(0, n-3)$.

Proof. The proof is similar to the proof of Proposition 27.

Proposition 35. With the above notations,
1) $\gamma(n, 0)$ are integers for all integer $n \geq 0$.
2) $\gamma(0, n)$ are integers for all integer $n \geq 0$.
3) $\mu(n, 0)$ are integers for all integer $n \geq 0$.
4) $\mu(0, n)$ are integers for all integer $n \geq 0$.

Proof. The proof is similar to the proof of Proposition 15. 
Theorem 36. For integers $i, j$,

$$
\left\{\left(\frac{\sqrt{7} \sin ^{i+j-1} 2 \theta}{2 \sin ^{i} 4 \theta \sin ^{j} 8 \theta}\right)^{n}+\left(\frac{\sqrt{7} \sin ^{i+j-1} 4 \theta}{2 \sin ^{i} 8 \theta \sin ^{j} 2 \theta}\right)^{n}+\left(\frac{\sqrt{7} \sin ^{i+j-1} 8 \theta}{2 \sin ^{i} 2 \theta \sin ^{j} 4 \theta}\right)^{n} \mid n=0,1,2, \cdots\right\}
$$

are integer sequences.

Proof. The proof is similar to the proof of Theorem 27.

Remark 37. Note that

$$
\frac{\sqrt{7} A^{i+j+1}}{2 B^{i} C^{j}} \cdot \frac{\sqrt{7} B^{i+j+1}}{2 C^{i} A^{j}} \cdot \frac{\sqrt{7} C^{i+j+1}}{2 A^{i} B^{j}}=-7
$$

By Proposition 41

$$
\left\{\frac{\sqrt{7} A^{i+j-1}}{2 B^{i} C^{j}}, \frac{\sqrt{7} B^{i+j-1}}{2 C^{i} A^{j}}, \frac{\sqrt{7} C^{i+j-1}}{2 A^{i} B^{j}}\right\}
$$

are the roots of a monic equation

$$
E Q(i, j)=t^{3}-\gamma(i, j) t^{2}+\mu(i, j) t+7=0 .
$$

Let $\operatorname{SE} Q(i, j)$ be the associated sequence.

Example 38. With the above notations,
1) $E Q(0,0)=t^{3}-7 t+7=0$.
$S E Q=3,0,14,-21,98,-245,833, \cdots \quad$ [Wang A275831]
2) $E Q(0,1)=t^{3}-7 t+7=0$.
$S E Q=3,0,14,-21,98,-245,833, \cdots$
3) $E Q(1,0)=t^{3}-7 t+7=0$.
$S E Q=3,0,14,-21,98,-245,833, \cdots$
4) $E Q(0,2)=t^{3}-7 t^{2}+7 t+7=0$.
$S E Q=3,7,35,175,931,5047,27587, \cdots \quad$ [Witula $\underline{\text { A215575] }}$
5) $E Q(1,1)=t^{3}+7 t^{2}+14 t+7=0$.
$S E Q=3,-7,21,-70,245,-882,3234, \cdots \quad$ [Wang A322459]
6) $E Q(2,0)=t^{3}-7 t^{2}+7=0$.
$S E Q=3,7,49,322,2205,15092,103390, \cdots$
7) $E Q(0,3)=t^{3}+7 t^{2}-28 t+7=0$.
$S E Q=3,-7,105,-952,9653,-94962,941682, \cdots$
8) $E Q(1,2)=t^{3}-21 t+7=0$.
$S E Q=3,0,42,-21,882,-735,18669, \cdots$
9) $E Q(2,1)=t^{3}-7 t^{2}-14 t+7=0$.
$S E Q=3,7,77,616,5341,45472,388766, \cdots$
10) $E Q(3,0)=t^{3}+14 t^{2}-21 t+7=0$.
$S E Q=3,-14,238,-3647,56154,-864409,13306489, \cdots$
11) $E Q(0,4)=t^{3}-21 t^{2}+56 t+7=0$.
$S E Q=3,21,329,5712,101381,1806826,32226026, \cdots$
12) $E Q(1,3)=t^{3}+14 t^{2}+49 t+7=0$.
$S E Q=3,-14,98,-707,5194,-38759,293069, \cdots$
13) $E Q(2,2)=t^{3}-14 t^{2}+35 t+7=0$. 


$$
S E Q=3,14,126,1253,13034,137739,1463385, \cdots
$$

14) $E Q(3,1)=t^{3}+21 t^{2}+35 t+7=0$.

$S E Q=3,-21,371,-7077,135779,-2606261,50028755, \cdots$

[Deleham $\underline{\mathrm{A} 108716]}$

15) $E Q(4,0)=t^{3}-35 t^{2}+14 t+7=0$. $S E Q=3,35,1197,41384,1431437,49512540,1712609094, \cdots$

\section{Power Sums of Cosine Values}

In this section we will compute the sum of powers and mixed powers of cosine values.

In this section and Section $8,9,10$, let

$$
A=\cos 2 \theta, B=\cos 4 \theta, C=\cos 8 \theta,
$$

The following result can be easily proved using equations in [4] [5].

Proposition 39. With the above notations, $\{A, B, C\}$ are the roots of the equation

$$
x^{3}+\frac{1}{2} x^{2}-\frac{1}{2} x-\frac{1}{8}=0 .
$$

$\{2 A, 2 B, 2 C\}$ are the roots of the equation

$$
x^{3}+x^{2}-2 x-1=0 .
$$

Definition 40. For an integer n,

$$
C(n)=A^{n}+B^{n}+C^{n} .
$$

For integers $i, j$, let

$$
V(i, j)=\sum A^{i} B^{j}
$$

Let

$$
E=A B C=\frac{1}{8}
$$

Proposition 41. With the above notations, $C(n)$ satisfy the following recurrence relations.

$$
\begin{gathered}
C(n)=-\frac{1}{2} C(n-1)+\frac{1}{2} C(n-2)+\frac{1}{8} C(n-3), \\
C(-n)=-4 C(-n+1)+4 C(-n+2)+8 C(-n+3) .
\end{gathered}
$$

Proof. The recurrence relations follow easily from the equations in Proposition 39.

Proposition 42. With the above notations, For $n=0, \cdots, 10$,

$$
\begin{gathered}
C(n)=3,-\frac{1}{2}, \frac{5}{4},-\frac{1}{2}, \frac{13}{16},-\frac{1}{2}, \frac{19}{32},-\frac{57}{128}, \frac{117}{256},-\frac{193}{512}, \frac{185}{512}, \cdots \\
C(-n)=3,-4,24,-88,416,-1824,8256,-36992,166400,-747520,3359744, \cdots
\end{gathered}
$$

Proposition 43. With above notations, $V(i, j)$ satisfy the following recurrence relations. 


$$
\begin{aligned}
& V(i, j)=-\frac{1}{2} V(i-1, j)+\frac{1}{2} V(i-2, j)+\frac{1}{8} V(i-3, j), \\
& V(i, j)=-\frac{1}{2} V(i, j-1)+\frac{1}{2} V(i, j-2)+\frac{1}{8} V(i, j-3) .
\end{aligned}
$$

Proof. The recurrence relations follow easily from the equations in Proposition 39.

Lemma 44. With the above notations, list of $V(i, j), i, j=1, \cdots, 8$

$$
\begin{aligned}
& \frac{-1}{2}, \frac{-1}{2}, \frac{-1}{16}, \frac{-9}{32}, \frac{3}{64}, \frac{-11}{64}, \frac{19}{256}, \frac{-15}{128}, \\
& \frac{3}{8}, \frac{3}{8}, \frac{5}{32}, \frac{5}{32}, \frac{3}{64}, \frac{19}{256}, \frac{3}{512}, \frac{41}{1024}, \\
& \frac{-1}{2}, \frac{-9}{32}, \frac{-11}{64}, \frac{-15}{128}, \frac{-1}{16}, \frac{-25}{512}, \frac{-11}{512}, \frac{-11}{512}, \\
& \frac{3}{8}, \frac{17}{64}, \frac{5}{32}, \frac{13}{128}, \frac{31}{512}, \frac{41}{1024}, \frac{47}{2048}, \frac{33}{2048}, \\
& \frac{-25}{64}, \frac{-29}{128}, \frac{-37}{256}, \frac{-23}{256}, \frac{-57}{1024}, \frac{-9}{256}, \frac{-11}{512}, \frac{-113}{8192}, \\
& \frac{41}{128}, \frac{27}{128}, \frac{33}{256}, \frac{83}{1024}, \frac{103}{2048}, \frac{129}{4096}, \frac{5}{256}, \frac{201}{16384}, \\
& \frac{-79}{256}, \frac{-95}{512}, \frac{-15}{128}, \frac{-149}{2048}, \frac{-93}{2048}, \frac{-29}{1024}, \frac{-289}{16384}, \frac{-361}{32768}, \\
& \frac{17}{64}, \frac{87}{512}, \frac{215}{2048}, \frac{269}{4096}, \frac{335}{8192}, \frac{209}{8192}, \frac{521}{32768}, \frac{325}{32768},
\end{aligned}
$$

Proof. Similar to the proof of Proposition 15.

\section{Alpha Sequences of Cosine Values}

In this section we will study the $\alpha$ sequences for cosine values.

Lemma 45

$$
Q=-4, R=3 \text {. }
$$

Proof. Recall that

$$
Q=\sum \frac{A}{B}, R=\sum \frac{A}{C}
$$

By Proposition 46

$$
\begin{gathered}
Q+R=C(1) C(-1)-3=\left(-\frac{1}{2}\right)(-4)-3=-1 . \\
Q R=\left(\frac{1}{E}\right) C(3)+(E) C(-3)+3=(8)\left(-\frac{1}{2}\right)+\left(\frac{1}{8}\right)(-4)+3=-12 .
\end{gathered}
$$

Then $\{Q, R\}=\{-4,3\}$ are the roots of the equation:

$$
t^{2}+t+12=0
$$

Note that $A \approx 0.782, B \approx 0.975, C \approx-0.434$. Then $Q \approx-4.0, R \approx 3.0$ It follows that $Q=-4, R=3$.

Proposition 46. With the above notations, $\left\{\frac{A}{B}, \frac{B}{C}, \frac{C}{A}\right\}$ are roots of equation 


$$
t^{3}+4 t^{2}-3 t-1=0
$$

Proof. By Lemma 45,

$$
\begin{gathered}
\frac{A}{B}+\frac{B}{C}+\frac{C}{A}=-4, \\
\frac{A}{B} \cdot \frac{B}{C}+\frac{B}{C} \cdot \frac{C}{A}+\frac{C}{A} \cdot \frac{A}{B}=\frac{A}{C}+\frac{B}{A}+\frac{C}{B}=3, \\
\frac{A}{B} \cdot \frac{B}{C} \cdot \frac{C}{A}=1,
\end{gathered}
$$

It follows that $\frac{A}{B}, \frac{B}{C}, \frac{C}{A}$ are the roots of the above equation.

\section{Proposition 47.}

$$
\alpha(0,0)=3, \alpha(1,0)=-4, \alpha(2,0)=10,
$$

and for $n \geq 0, \alpha(n, 0)$ satisfies recurrence equation:

$$
\begin{gathered}
\alpha(n, 0)=-4 \alpha(n-1,0)+3 \alpha(n-2,0)+\alpha(n-3,0) . \\
\alpha(0,0)=3, \alpha(0,1)=3, \alpha(0,2)=17,
\end{gathered}
$$

and for $n \geq 0, \alpha(0, n)$ satisfies recurrence equation:

$$
\alpha(0, n)=-3 \alpha(0, n-1)+4 \alpha(0, n-2)+\alpha(0, n-3) .
$$

Proof. Recall that

$$
\alpha(i, j)=\sum \frac{A^{i+j}}{B^{i} C^{j}} .
$$

The proof is similar to Proposition 19,

Theorem 48.

$$
\left\{\left(\frac{\cos 2 \theta}{\cos 4 \theta}\right)^{n}+\left(\frac{\cos 4 \theta}{\cos 8 \theta}\right)^{n}+\left(\frac{\cos 8 \theta}{\cos 2 \theta}\right)^{n} \mid n=0, \pm 1, \pm 2, \cdots\right\}
$$

is a two-sided integer sequence.

Proof. The proof is similar to Theorem 19.

Example 49. For $n=0,1,2,3, \cdots$

$\{\alpha(n, 0) \mid n=0,1,2,3, \cdots\}=\{3,-4,10,-25,66,-179,493,-1369,3818,-10672, \cdots\} \quad$ [Wang $\underline{\text { A274220 }}]$ $\{\alpha(0, n) \mid n=0,1,2,3, \cdots\}=\{3,3,17,66,269,1088,4406,17839,72229,292449, \cdots\} \quad$ [Witula A215076]

\section{Lemma 50.}

$$
\alpha(1,1)=-4, \alpha(-1,-1)=-11
$$

Proof.

$$
\begin{gathered}
\alpha(1,1)=\sum \frac{A^{2}}{B C}=\frac{1}{E} \sum A^{3}=(8)\left(-\frac{1}{2}\right)=-4, \\
\alpha(-1,-1)=\sum \frac{B C}{A^{2}}=E \sum \frac{1}{A^{3}}=\left(\frac{1}{8}\right)(-4)=-11 .
\end{gathered}
$$

Theorem 51. For integers $i, j$, 


$$
\left\{\left(\frac{\cos ^{i+j} 2 \theta}{\cos ^{i} 4 \theta \cos ^{j} 8 \theta}\right)^{n}+\left(\frac{\cos ^{i+j} 4 \theta}{\cos ^{i} 8 \theta^{i} \cos ^{j} 2 \theta}\right)^{n}+\left(\frac{\cos ^{i+j} 8 \theta}{\cos ^{i} 2 \theta \cos ^{j} 4 \theta}\right)^{n} \mid n=0, \pm 1, \pm 2, \cdots\right\}
$$

is a two-sided integer sequence.

Proof. The proof is similar to Theorem 23.

Remark 52. using our approach, we can generate two-sided integer sequences as follows.

1) For each $(i, j), i \geq 0, j \geq 0$, we can compute $\alpha(i, j), \alpha(-i,-j)$ using recurrence relations in Proposition 47 and Proposition 6.

2) Let

$$
X=\frac{A^{i+j}}{B^{i} C^{j}}, Y=\frac{B^{i+j}}{C^{i} A^{j}}, Z=\frac{C^{i+j}}{A^{i} B^{j}} .
$$

Then $\{X, Y, Z\}$ are the roots for equation:

$$
t^{3}-\alpha(i, j) t^{2}+\alpha(-i,-j) t-1=0 .
$$

3) Let $p(n)=\sum X^{n}$. Then

$$
p(0)=3, p(1)=\alpha(i, j), p(2)=\alpha(i, j)^{2}-2 \alpha(-i,-j) .
$$

4) For $n \geq 3$ and $n<0$ use the recurrence relation

$$
p(n)=\alpha(i, j) p(n-1)-\alpha(-i,-j) p(n-2)+p(n-3)
$$

to get $p(n)$.

\section{Remark 53. Note that}

1) Integer sequence for $(n, 0)$ is a subsequence for the integer sequence for $(1,0)$.

2) Integer sequence for $(0, n)$ is a subsequence for the integer sequence for $(0, n)$.

\section{Example 54}

1) $E Q(0,0)=t^{3}-3 t^{2}+3 t-1=0$.

$S E Q 1=3,3,3,3,3,3,3, \cdots$

$S E Q 2=3,3,3,3,3,3,3, \cdots$

2) $E Q(0,1)=t^{3}-3 t^{2}-4 t-1=0$.

$S E Q 1=3,3,17,66,269,1088,4406, \cdots \quad$ [Witula A215076]

$S E Q 2=3,-4,10,-25,66,-179,493, \cdots \quad$ [Wang A274220]

3) $E Q(1,0)=t^{3}+4 t^{2}+3 t-1=0$.

$S E Q 1=3,-4,10,-25,66,-179,493, \cdots \quad$ [Wang A274220]

$S E Q 2=3,3,17,66,269,1088,4406, \cdots \quad$ [Witula A215076]

4) $E Q(0,2)=t^{3}-17 t^{2}+10 t-1=0$.

$S E Q 1=3,17,269,4406,72229,1184102,19411850, \cdots$

$S E Q 2=3,10,66,493,3818,29865,234237, \cdots$

5) $E Q(1,1)=t^{3}+4 t^{2}-11 t-1=0$.

$S E Q 1=3,-4,38,-193,1186,-6829,40169, \cdots \quad$ [Wang A274663]

$S E Q 2=3,-11,129,-1460,16565,-187926,2131986, \cdots$

6) $E Q(2,0)=t^{3}-10 t^{2}+17 t-1=0$. 
$S E Q 1=3,10,66,493,3818,29865,234237, \cdots$

$S E Q 2=3,17,269,4406,72229,1184102,19411850, \cdots$

7) $E Q(0,3)=t^{3}-66 t^{2}-25 t-1=0$.

$S E Q 1=3,66,4406,292449,19411850,1288497731,85526438945, \cdots$

$S E Q 2=3,-25,493,-10672,234237,-5151080,113306686, \cdots$

8) $E Q(1,2)=t^{3}+25 t^{2}+31 t-1=0$.

$S E Q 1=3,-25,563,-13297,314947,-7460905,176745971, \cdots$

$S E Q 2=3,31,1011,32119,1020995,32454831,1031656755, \cdots$

9) $E Q(2,1)=t^{3}-3 t^{2}-46 t-1=0$.

$S E Q 1=3,3,101,444,5981,38468,390974, \cdots \quad$ [Witula A215560]

$S E Q 2=3,-46,2110,-96919,4451898,-204494441,9393291673, \cdots$

10) $E Q(3,0)=t^{3}+25 t^{2}+66 t-1=0$.

$S E Q 1=3,-25,493,-10672,234237,-5151080,113306686, \cdots$

$S E Q 2=3,66,4406,292449,19411850,1288497731,85526438945, \cdots$

11) $E Q(0,4)=t^{3}-269 t^{2}+66 t-1=0$.

$S E Q 1=3,269,72229,19411850,5217020805$,

$1402097486674,376819919954026, \cdots$

$S E Q 2=3,66,3818,234237,14432666,889550021,54828148469, \cdots$

12) $E Q(1,3)=t^{3}+95 t^{2}-88 t-1=0$.

$S E Q 1=3,-95,9201,-882452,84642533,-8118687210,778722945402, \cdots$

[Wang A322460]

$S E Q 2=3,-88,7934,-706549,62929954,-5604950173,499213254305, \cdots$

13) $E Q(2,2)=t^{3}-38 t^{2}+129 t-1=0$.

$S E Q 1=3,38,1186,40169,1373466,47011093,1609284589, \cdots$

$S E Q 2=3,129,16565,2131986,274396853,35316195134$,

$4545364223858, \cdots$

14) $E Q(3,1)=t^{3}-3 t^{2}-186 t-1=0$.

SEQ1 $=3,3,381,1704,75981,545268,15769974, \cdots$

$S E Q 2=3,-186,34590,-6433179,1196467338$,

$-222523590741,41385792042633, \cdots$

15) $E Q(4,0)=t^{3}-66 t^{2}+269 t-1=0$.

$S E Q 1=3,66,3818,234237,14432666,889550021,54828148469, \cdots$

$S E Q 2=3,269,72229,19411850,5217020805$,

$1402097486674,376819919954026, \cdots$

\section{Beta Sequences of Cosine Values}

In this section we will study the $\beta$ sequences for cosine values. Let $K=2$ in the formula for $\beta(i, j)$ and $\lambda(i, j)$. Then

$$
\beta(i, j)=\sum \frac{2 A^{i+j+1}}{B^{i} C^{j}}, \lambda(i, j)=\sum \frac{4 B^{i+1} C^{j+1}}{A^{i+j}} .
$$

Lemma 55. With above notations,

1) $\beta(0,0)=-1$,

2) $\beta(1,0)=-1$, 

3) $\beta(0,1)=-8$,
4) $\beta(2,0)=6$,
5) $\beta(0,2)=-29$,
6) $\beta(1,1)=13$,
7) $\lambda(0,0)=-2$,
8) $\lambda(1,0)=-9$,
9) $\lambda(0,1)=5$,
10) $\lambda(2,0)=-37$,
11) $\lambda(0,2)=-16$,
12) $\lambda(1,1)=26$.

Proof. To prove (1),

$$
\beta(0,0)=2 \sum A=2 \cdot \frac{-1}{2}=-1 .
$$

To prove (2),

$$
\beta(1,0)=2 \sum \frac{A^{2}}{B}=\frac{2}{E} \sum A B^{3}=\frac{2}{E} V(1,3)=-1 .
$$

To prove (3),

$$
\beta(0,1)=2 \sqrt{7} \sum \frac{A^{2}}{C}=\frac{2}{E} \sum A^{3} B=\frac{2}{E} V(3,1)=-8 .
$$

To prove (4),

$$
\beta(2,0)=2 \sum \frac{A^{3}}{B^{2}}=\frac{2}{E^{2}} \sum A^{2} B^{5}=\frac{2}{E^{2}} V(2,5)=6 .
$$

To prove (5),

$$
\beta(0,2)=2 \sum \frac{A^{3}}{C^{2}}=\frac{2}{E^{2}} \sum A^{5} B^{2}=\frac{2}{E^{2}} V(5,2)=-29 .
$$

To prove (6),

$$
\beta(1,1)=2 \sum \frac{A^{3}}{B C}=\frac{2}{E} \sum A^{4}=\frac{2}{E} C(4)=13 .
$$

To prove (7),

$$
\lambda(0,0)=4 \sum B C=4 E \sum \frac{1}{A}=4 E C(-1)=-2 .
$$

To prove (8),

$$
\lambda(1,0)=4 \sum \frac{B^{2} C}{A}=\frac{4}{P} \sum A^{3} B^{2}=\frac{4}{E} V(3,2)=-9 .
$$

To prove (9),

$$
\lambda(0,1)=4 \sum \frac{B C^{2}}{A}=\frac{4}{E} \sum A^{2} B^{3}=\frac{4}{E} V(2,3)=5 .
$$

To prove (10),

$$
\lambda(2,0)=4 \sum \frac{B^{3} C}{A^{2}}=\frac{4}{E^{2}} \sum A^{5} B^{3}=\frac{4}{E^{2}} V(5,3)=-37 .
$$


To prove (11),

$$
\lambda(0,2)=4 \sum \frac{B C^{3}}{A^{2}}=\frac{4}{E^{2}} \sum A^{3} B^{5}=\frac{4}{E^{2}} V(3,5)=-16 .
$$

To prove (12),

$$
\lambda(1,1)=4 \sum \frac{B^{2} C^{2}}{A^{2}}=4 E^{2} \sum \frac{1}{A^{4}}=4 E^{2} C(-4)=26 .
$$

Proposition 56. With the above notations,

1) $\beta(n, 0)=-4 \beta(n-1,0)+3 \beta(n-2,0)+\beta(n-3,0)$.

2) $\beta(0, n)=-3 \beta(0, n-1)+4 \beta(0, n-2)+\beta(0, n-3)$,

3) $\lambda(n, 0)=-3 \lambda(n-1,0)+4 \lambda(n-2,0)+\lambda(n-3,0)$,

4) $\lambda(0, n)=-4 \lambda(0, n-1)+3 \lambda(0, n-2)+\lambda(0, n-3)$.

Proof. By Proposition 46, $\left\{\frac{A}{B}, \frac{B}{C}, \frac{C}{A}\right\}$ are roots of equation

$$
t^{3}+4 t^{2}-3 t-1=0 \text {. }
$$

The rest of the proof is similar to the proof for Proposition 28.

Proposition 57. With the above notations,

1) $\beta(n, 0)$ are integers for all integer $n \geq 0$,

2) $\beta(0, n)$ are integers for all integer $n \geq 0$,

3) $\lambda(n, 0)$ are integers for all integer $n \geq 0$,

4) $\lambda(0, n)$ are integers for all integer $n \geq 0$.

Proof. The rest of the proof is similar to the proof for Proposition 29.

Theorem 58. For integers $i, j$,

$$
\left\{\left(\frac{2 \sqrt{7} \cos ^{i+j+1} 2 \theta}{\cos ^{i} 4 \theta \cos ^{j} 8 \theta}\right)^{n}+\left(\frac{2 \sqrt{7} \cos ^{i+j+1} 4 \theta}{\cos ^{i} 8 \theta \cos ^{j} 2 \theta}\right)^{n}+\left(\frac{2 \sqrt{7} \cos ^{i+j+1} 8 \theta}{\cos ^{i} 2 \theta \cos ^{j} 4 \theta}\right)^{n} \mid n=0,1,2, \cdots\right\}
$$

are integer sequences.

Proof. The proof is similar to the proof for Theorem 30.

Remark 59. Note that

$$
\frac{2 A^{i+j+1}}{B^{i} C^{j}} \cdot \frac{2 B^{i+j+1}}{C^{i} A^{j}} \cdot \frac{2 C^{i+j+1}}{A^{i} B^{j}}=1 .
$$

By Proposition $5\left\{\frac{2 A^{i+j+1}}{B^{i} C^{j}}, \frac{2 B^{i+j+1}}{C^{i} A^{j}}, \frac{2 C^{i+j+1}}{A^{i} B^{j}}\right\}$ are the roots of a monic equation

$$
E Q(i, j)=t^{3}-\beta(i, j) t^{2}+\lambda(i, j) t-1=0 .
$$

Let $\operatorname{SEQ}(i, j)$ be the associated sequence.

Example 60. With the above notations,

1) $E Q(0,0)=t^{3}+t^{2}-2 t-1=0$.

$S E Q 1=3,-1,5,-4,13,-16,38, \cdots \quad$ [Barry A094648]

$S E Q 2=3,-2,6,-11,26,-57,129, \cdots \quad[$ Wang A274975]

2) $E Q(0,1)=t^{3}+8 t^{2}+5 t-1=0$.

$S E Q 1=3,-8,54,-389,2834,-20673,150825, \cdots$

$S E Q 2=3,5,41,248,1573,9890,62282, \cdots$ 
3) $E Q(1,0)=t^{3}+t^{2}-9 t-1=0$.

$S E Q 1=3,-1,19,-25,195,-401,2131, \cdots \quad$ [Wang A274075]

$S E Q 2=3,-9,83,-753,6851,-62329,567059, \cdots \quad$ [Wang A274032]

4) $E Q(0,2)=t^{3}+29 t^{2}-16 t-1=0$.

$S E Q 1=3,-29,873,-25778,761501,-22495104,664516254, \cdots$

$S E Q 2=3,-16,314,-5485,96850,-1708351,30136781, \cdots$

5) $E Q(1,1)=t^{3}-13 t^{2}+26 t-1=0$.

$S E Q 1=3,13,117,1186,12389,130338,1373466, \cdots$

$S E Q 2=3,26,650,16565,422266,10764221,274396853, \cdots$

6) $E Q(2,0)=t^{3}-6 t^{2}-37 t-1=0$.

$S E Q 1=3,6,110,885,9386,89171,883193, \cdots$

$S E Q 2=3,-37,1357,-49984,1841229,-67824212,2498398486, \cdots$

7) $E Q(0,3)=t^{3}+120 t^{2}+47 t-1=0$.

$S E Q 1=3,-120,14306,-1711077,204656738$,

$-24478373635,2927784258437, \cdots$

$S E Q 2=3,47,2449,120746,5968989,295034452,14583018670, \cdots$

8) $E Q(1,2)=t^{3}-41 t^{2}-72 t-1=0$.

$S E Q 1=3,41,1825,77780,3320421,141739246,6050457178, \cdots$

$S E Q 2=3,-72,5102,-364389,26026754,-1858981237,132779187761, \cdots$

9) $E Q(2,1)=t^{3}+22 t^{2}+103 t-1=0$.

$S E Q 1=3,-22,278,-3847,55978,-834997,12600353, \cdots$

$S E Q 2=3,103,10653,1099528,113485853,11713243128,1208961830478, \cdots$

10) $E Q(3,0)=t^{3}+22 t^{2}-149 t-1=0$.

$S E Q 1=3,-22,782,-20479,567034,-15525337,426025001, \cdots$

SEQ2 $=3,-149,22245,-3317780,494838461$,

$-73803899604,11007664169358, \cdots$

11) $E Q(0,4)=t^{3}+484 t^{2}-135 t-1=0$.

$S E Q 1=3,-484,234526,-113575921,55002406290$, $-26636497159169,12899489836311025, \cdots$

SEQ2 = 3,-135,19193,-2656392,367902197, $-50952471130,7056645609506, \cdots$

12) $E Q(1,3)=t^{3}-174 t^{2}+201 t-1=0$.

$S E Q 1=3,174,29874,5163105,892375770$, $154235629749,26657637209661, \cdots$

$S E Q 2=3,201,40053,8015682,1604183061$, $321046106646,64251147598914, \cdots$

13) $E Q(2,2)=t^{3}+57 t^{2}-289 t-1=0$.

$S E Q 1=3,-57,3827,-234609,14478659,-893081737,55089756851, \cdots$

$S E Q 2=3,-289,83635,-24186985,6994805571$, $-2022877384529,585010243859443, \cdots$

14) $E Q(3,1)=t^{3}-41 t^{2}+418 t-1=0$.

$S E Q 1=3,41,845,17510,364741,7636046,160633658, \cdots$

$S E Q 2=3,418,174642,72983221,30499826474$, $12745935328713,5326550547499821, \cdots$ 
15) $E Q(4,0)=t^{3}-69 t^{2}-604 t-1=0$.

$S E Q 1=3,69,5969,453540,34899605,2682016874,206138979266, \cdots$

$S E Q 2=3,-604,364678,-220223833,132990031746$,

$-80310783365429,48498536620304809, \cdots$

\section{Gamma Sequences of Cosine Values}

In this section we will study the $\gamma$ sequences for cosine values. Let $K=\frac{1}{2}$ in the formula for $\gamma(i, j)$ and $\mu(i, j)$. Then

$$
\gamma(i, j)=\sum \frac{A^{i+j-1}}{2 B^{i} C^{j}}, \mu(i, j)=\sum \frac{B^{i-1} C^{j-1}}{4 A^{i+j}} .
$$

Lemma 61. With the above notations,

1) $\gamma(0,0)=-2$,

2) $\gamma(1,0)=-2$,

3) $\gamma(0,1)=-2$,

4) $\gamma(2,0)=5$,

5) $\gamma(0,2)=-9$,

6) $\gamma(1,1)=5$

7) $\mu(0,0)=-1$,

8) $\mu(1,0)=-1$,

9) $\mu(0,1)=-1$,

10) $\mu(2,0)=-8$,

11) $\mu(0,2)=-1$,

12) $\mu(1,1)=6$.

Proof. The proof is similar to the proof of Lemma 33.

Proposition 62. With above notations,

1) $\gamma(n, 0)=-4 \gamma(n-1,0)+3 \gamma(n-2,0)+\gamma(n-3,0)$,

2) $\gamma(0, n)=-3 \gamma(0, n-1)+4 \gamma(0, n-2)+\gamma(0, n-3)$,

3) $\mu(n, 0)=-3 \mu(n-1,0)+4 \mu(n-2,0)+\mu(n-3,0)$,

4) $\mu(0, n)=-4 \mu(0, n-1)+3 \mu(0, n-2)+\mu(0, n-3)$.

Proof. The proof is similar to the proof of Proposition 34.

Proposition 63. With above notations,

1) $\gamma(n, 0)$ are integers for all integer $n \geq 0$.

2) $\gamma(n, 0)$ are integers for all integer $n \geq 0$.

3) $\mu(n, 0)$ are integers for all integer $n \geq 0$.

4) $\mu(0, n)$ are integers for all integer $n \geq 0$.

Proof. The proof is similar to the proof of Proposition 35.

Theorem 64. For integers $i, j$,

$\left\{\left(\frac{\cos ^{i+j-1} 2 \theta}{2 \cos ^{i} 4 \theta \cos ^{j} 8 \theta}\right)^{n}+\left(\frac{\cos ^{i+j-1} 4 \theta}{2 \cos ^{i} 8 \theta \cos ^{j} 2 \theta}\right)^{n}+\left(\frac{\cos ^{i+j-1} 8 \theta}{2 \cos ^{i} 2 \theta \cos ^{j} 4 \theta}\right)^{n} \mid n=0,1,2, \cdots\right\}$

are integer sequences.

Proof. The proof is similar to the proof of Proposition 36. 
Remark 65. Note that

$$
\frac{A^{i+j-1}}{2 B^{i} C^{j}} \cdot \frac{B^{i+j-1}}{2 C^{i} A^{j}} \cdot \frac{C^{i+j-1}}{2 A^{i} B^{j}}=1 .
$$

By Proposition $41\left\{\frac{A^{i+j-1}}{2 B^{i} C^{j}}, \frac{B^{i+j-1}}{2 C^{i} A^{j}}, \frac{C^{i+j-1}}{2 A^{i} B^{j}}\right\}$ are the roots of a monic equation

$$
E Q(i, j)=t^{3}-\gamma(i, j) t^{2}+\mu(i, j) t-1=0 .
$$

Let $\operatorname{SEQ}(i, j)$ be the associated sequence.

Example 66. List of sequences:

1) $E Q(0,0)=t^{3}+2 t^{2}-t-1=0$.

$S E Q 1=3,-2,6,-11,26,-57,129, \cdots \quad$ [Wang A274975, Sloane A033304]

$S E Q 2=3,-1,5,-4,13,-16,38, \cdots \quad$ [Barry A094648, A096975]

2) $E Q(0,1)=t^{3}+2 t^{2}-t-1=0$.

$S E Q 1=3,-2,6,-11,26,-57,129, \cdots$

$S E Q 2=3,-1,5,-4,13,-16,38, \cdots$

3) $E Q(1,0)=t^{3}+2 t^{2}-t-1=0$.

$S E Q 1=3,-2,6,-11,26,-57,129, \cdots$

$S E Q 2=3,-1,5,-4,13,-16,38, \cdots$

4) $E Q(0,2)=t^{3}+9 t^{2}-t-1=0$.

$S E Q 1=3,-9,83,-753,6851,-62329,567059, \cdots \quad$ [Wang A274032]

$S E Q 2=3,-1,19,-25,195,-401,2131, \cdots \quad$ [Wang $\underline{\text { A274075 }}]$

5) $E Q(1,1)=t^{3}-5 t^{2}+6 t-1=0$.

$S E Q 1=3,5,13,38,117,370,1186, \cdots \quad$ [Lang A198636]

$S E Q 2=3,6,26,129,650,3281,16565, \cdots$

6) $E Q(2,0)=t^{3}-5 t^{2}-8 t-1=0$.

$S E Q 1=3,5,41,248,1573,9890,62282, \cdots$

$S E Q 2=3,-8,54,-389,2834,-20673,150825, \cdots$

7) $E Q(0,3)=t^{3}+37 t^{2}+6 t-1=0$.

$S E Q 1=3,-37,1357,-49984,1841229,-67824212,2498398486, \cdots$

$S E Q 2=3,6,110,885,9386,89171,883193, \cdots$

8) $E Q(1,2)=t^{3}-12 t^{2}-15 t-1=0$.

$S E Q 1=3,12,174,2271,29874,392727,5163105, \cdots$

$S E Q 2=3,-15,201,-2832,40053,-566610,8015682, \cdots$

9) $E Q(2,1)=t^{3}+9 t^{2}+20 t-1=0$.

SEQ1 $=3,-9,41,-186,845,-3844,17510, \cdots \quad$ [Wang A320918]

$S E Q 2=3,20,418,8543,174642,3570145,72983221, \cdots \quad$ [Wang A322455]

10) $E Q(3,0)=t^{3}+16 t^{2}-29 t-1=0$.

$S E Q 1=3,-16,314,-5485,96850,-1708351,30136781, \cdots$

$S E Q 2=3,-29,873,-25778,761501,-22495104,664516254, \cdots$

11) $E Q(0,4)=t^{3}+149 t^{2}-22 t-1=0$.

$S E Q 1=3,-149,22245,-3317780,494838461$,

$-73803899604,11007664169358, \cdots$

SEQ2 $=3,-22,782,-20479,567034,-15525337,426025001, \cdots$ 
12) $E Q(1,3)=t^{3}-54 t^{2}+41 t-1=0$.

$S E Q 1=3,54,2834,150825,8028410,427353149,22748056061, \cdots$

$S E Q 2=3,41,1573,62282,2468661,97853446,3878745874, \cdots$

13) $E Q(2,2)=t^{3}+16 t^{2}-57 t-1=0$.

$S E Q 1=3,-16,370,-6829,130338,-2474291,47011093, \cdots$

$S E Q 2=3,-57,3281,-187926,10764221,-616564132,35316195134, \cdots$

14) $E Q(3,1)=t^{3}-19 t^{2}+83 t-1=0$.

$S E Q 1=3,19,195,2131,24323,285459,3407043, \cdots$

$S E Q 2=3,83,6851,567059,46935811,3884905043,321555905219, \cdots$

15) $E Q(4,0)=t^{3}-47 t^{2}-120 t-1=0$.

$S E Q 1=3,47,2449,120746,5968989,295034452,14583018670, \cdots$

$S E Q 2=3,-120,14306,-1711077,204656738$,

$-24478373635,2927784258437, \cdots$

\section{Power Sums of Tangent Values}

In this section we will compute the sum of powers and mixed powers of tangent values.

In this section and Section 12, 13, 14, let

$$
A=\tan 2 \theta, B=\tan 4 \theta, C=\tan 8 \theta,
$$

The following result can be easily proved using equations in [4] [5].

Proposition 67. With the above notations, $\{A, B, C\}$ are the roots of the equation

$$
x^{3}+\sqrt{7} x^{2}-7 x+\sqrt{7}=0 .
$$

$\left\{A^{2}, B^{2}, C^{2}\right\}$ are the roots of the equation

$$
x^{3}-21 x^{2}+35 x-7=0
$$

Definition 68. For an integer $n$,

$$
T(n)=A^{n}+B^{n}+C^{n} .
$$

For integers $i, j$, let

$$
W(i, j)=\sum A^{i} B^{j}
$$

Let

Proposition 69. With the above notations, $T(n)$ satisfy the following recurrence relations.

$$
\begin{gathered}
T(n)=-\sqrt{7} T(n-1)+7 T(n-2)-\sqrt{7} T(n-3), \\
T(-n)=\sqrt{7} T(-n+1)-T(-n+2)-\frac{\sqrt{7}}{7} T(-n+3) .
\end{gathered}
$$

Proof. The recurrence relations follow easily from the equations in Proposition 67.

Example 70. With the above notations, For $n=0, \cdots, 10$,

$$
\begin{aligned}
T(n)= & 3,-\sqrt{7}, 7 \cdot 3,-31 \sqrt{7}, 7 \cdot 53,-7 \cdot 87 \sqrt{7}, 7 \cdot 1011,-7^{2} \cdot 239 \sqrt{7}, \\
& 7^{2} \cdot 2771,-7 \cdot 32119 \sqrt{7}, 7^{2} \cdot 53189, \cdots
\end{aligned}
$$




$$
T(-n)=3, \sqrt{7}, 5, \frac{25 \sqrt{7}}{7}, 19, \frac{103 \sqrt{7}}{7}, \frac{563}{7}, 7 \cdot 9 \sqrt{7}, \frac{2421}{7}, \frac{13297 \sqrt{7}}{7^{2}}, \frac{10435}{7}, \cdots
$$

Proposition 71. With the above notations, $W(i, j)$ satisfy the following recurrence relations.

$$
\begin{aligned}
& W(i, j)=-\sqrt{7} W(i-1, j)+7 W(i-2, j)-\sqrt{7} W(i-3, j), \\
& W(i, j)=-\sqrt{7} W(i, j-1)+7 W(i, j-2)-\sqrt{7} W(i, j-3) .
\end{aligned}
$$

Lemma 72. With above notations, list of $W(i, j), i, j=0,1, \cdots, 6$

$$
\begin{aligned}
& -7, \quad 9 \sqrt{7}, \quad-105,175 \sqrt{7},-2023,3353 \sqrt{7} \text {, } \\
& \sqrt{7}, \quad 35, \quad-49 \sqrt{7}, 581,-959 \sqrt{7}, 11123 \text {, } \\
& -49, \quad 7 \sqrt{7}, \quad-175,273 \sqrt{7},-3185,5271 \sqrt{7} \text {, } \\
& 63 \sqrt{7}, \quad 133,-63 \sqrt{7}, 931,-1505 \sqrt{7}, 17493 \text {, } \\
& -791,-119 \sqrt{7},-441,399 \sqrt{7},-5047,8281 \sqrt{7} \text {, } \\
& 1281 \sqrt{7}, 1715,175 \sqrt{7}, 1813,-2303 \sqrt{7}, 27587 .
\end{aligned}
$$

Proof. The proof is similar to the proof of Proposition 15.

\section{Alpha Sequences of Tangent Values}

In this section we will study the $\alpha$ sequences for tangent values.

Lemma 73.

$$
Q=-9, R=-1 \text {. }
$$

Proof. Recall that

$$
Q=\sum \frac{A}{B}, R=\sum \frac{A}{C}
$$

By Proposition 9

$$
\begin{gathered}
Q+R=T(1) T(-1)-3=(-\sqrt{7})(\sqrt{7})-3=-10 . \\
Q R=\left(\frac{1}{F}\right) T(3)+(F) T(-3)+3=9 .
\end{gathered}
$$

Then $\{Q, R\}=\{-1,-9\}$ are the roots of the equation:

$$
t^{2}+10 t+9=0 .
$$

Note that $A \approx 0.782, B \approx 0.975, C \approx-0.434$. Then $Q \approx-9.0, R \approx-1.0$ It follows that $Q=-9, R=-1$.

Proposition 74. With the above notations, $\left\{\frac{A}{B}, \frac{B}{C}, \frac{C}{A}\right\}$ are roots of equation

$$
t^{3}+9 t^{2}-t-1=0
$$

Proof. By Lemma 73,

$$
\begin{gathered}
\frac{A}{B}+\frac{B}{C}+\frac{C}{A}=-9, \\
\frac{A}{B} \cdot \frac{B}{C}+\frac{B}{C} \cdot \frac{C}{A}+\frac{C}{A} \cdot \frac{A}{B}=\frac{A}{C}+\frac{B}{A}+\frac{C}{B}=-1,
\end{gathered}
$$




$$
\frac{A}{B} \cdot \frac{B}{C} \cdot \frac{C}{A}=1
$$

It follows that $\frac{A}{B}, \frac{B}{C}, \frac{C}{A}$ are the roots of the above equation.

Proposition 75.

$$
\alpha(0,0)=3, \alpha(1,0)=-9, \alpha(2,0)=83
$$

and for $n \geq 0, \alpha(n, 0)$ satisfies recurrence equation:

$$
\begin{gathered}
\alpha(n, 0)=a-5 \alpha(n-1,0)+\alpha(n-2,0)+\alpha(n-3,0) . \\
\alpha(0,0)=3, \alpha(0,1)=-1, \alpha(0,2)=19
\end{gathered}
$$

and for $n \geq 0, \alpha(0, n)$ satisfies recurrence equation:

$$
\alpha(0, n)=-\alpha(0, n-1)+9 \alpha(0, n-2)+\alpha(0, n-3) .
$$

Proof. Recall that

$$
\alpha(i, j)=\sum \frac{A^{i+j}}{B^{i} C^{j}} .
$$

Then the proof is similar to the proof of Proposition 19.

Theorem 76. For integers $i, j$,

$$
\left\{\left(\frac{\tan 2 \theta}{\tan 4 \theta}\right)^{n}+\left(\frac{\tan 4 \theta}{\tan 8 \theta}\right)^{n}+\left(\frac{\tan 8 \theta}{\tan 2 \theta}\right)^{n} \mid n=0, \pm 1, \pm 2, \cdots\right\}
$$

is a two-sided integer sequence.

Proof. The proof is similar to the proof of Theorem 19.

Example 77. List of associated sequences:

1) $\quad\{\alpha(n, 0) \mid n=0,1,2,3, \cdots\}=\{3,-9,83,-753,6851,-62329, \cdots\} \quad$ [Wang A274032]

2) $\quad\{\alpha(0, n) \mid n=0,1,2,3, \cdots\}=\{3,-1,19,-25,195,-401,2131, \cdots\} \quad$ [Wang $\underline{\mathrm{A} 274075}$

Lemma 78.

$$
\alpha(1,1)=31, \alpha(-1,-1)=-25 \text {. }
$$

Proof.

$$
\begin{gathered}
\alpha(1,1)=\sum \frac{A^{2}}{B C}=\frac{1}{F} \sum A^{3}=\frac{1}{-\sqrt{7}} T(3)=31, \\
\alpha(-1,-1)=\sum \frac{B C}{A^{2}}=F \sum \frac{1}{A^{3}}=(-\sqrt{7}) T(-3)=-25 .
\end{gathered}
$$

Theorem 79. For integers $i, j$,

$$
\left\{\left(\frac{\tan ^{i+j} 2 \theta}{\tan ^{i} 4 \theta \tan ^{j} 8 \theta}\right)^{n}+\left(\frac{\tan ^{i+j} 4 \theta}{\tan ^{i} 8 \theta \tan ^{j} 2 \theta}\right)^{n}+\left(\frac{\tan ^{i+j} 8 \theta}{\tan ^{i} 2 \theta \tan ^{j} 4 \theta}\right)^{n} \mid n=0, \pm 1, \pm 2, \cdots\right\}
$$

is a two-sided integer sequence.

Proof. The proof is similar to the proof of Theorem 23.

Remark 80. Using our approach we can generate two-sided integer sequences 
as follows:

1) For each $(i, j), i \geq 0, j \geq 0$, we can compute $\alpha(i, j), \alpha(-i,-j)$ using recurrence relations in Proposition 23.

2) Let

$$
X=\frac{A^{i+j}}{B^{i} C^{j}}, Y=\frac{B^{i+j}}{C^{i} A^{j}}, Z=\frac{C^{i+j}}{A^{i} B^{j}} .
$$

Then $\{X, Y, Z\}$ are the roots for equation:

$$
t^{3}-\alpha(i, j) t^{2}+\alpha(-i,-j) t-1=0 .
$$

3) Let $p(n)=\sum X^{n}$. Then

$$
p(0)=3, p(1)=\alpha(i, j), p(2)=\alpha(i, j)^{2}-2 \alpha(-i,-j) .
$$

4) For $n \geq 3$ and $n<0$ use the recurrence relation

$$
p(n)=\alpha(i, j) p(n-1)-\alpha(-i,-j) p(n-2)+p(n-3)
$$

to get $p(n)$.

\section{Remark 81. Note that}

1) Integer sequence for $(n, 0)$ is a subsequence for the integer sequence for $(1,0)$.

2) Integer sequence for $(0, n)$ is a subsequence for the integer sequence for $(0,1)$.

Example 82. List of associated sequences.

1) $E Q(0,0)=t^{3}-3 t^{2}+3 t-1=0$.

$S E Q 1=3,3,3,3,3,3,3, \cdots$

$S E Q 2=3,3,3,3,3,3,3, \cdots$

2) $E Q(0,1)=t^{3}+t^{2}-9 t-1=0$.

SEQ1 $=3,-1,19,-25,195,-401,2131, \cdots \quad$ [Wang A274075]

$S E Q 2=3,-9,83,-753,6851,-62329,567059, \cdots \quad$ [Wang A274032]

3) $E Q(1,0)=t^{3}+9 t^{2}-t-1=0$.

$S E Q 1=3,-9,83,-753,6851,-62329,567059, \cdots \quad$ [Wang A274032]

$S E Q 2=3,-1,19,-25,195,-401,2131, \cdots \quad$ [Wang A274075]

4) $E Q(0,2)=t^{3}-19 t^{2}+83 t-1=0$.

$S E Q 1=3,19,195,2131,24323,285459,3407043, \cdots$

$S E Q 2=3,83,6851,567059,46935811,3884905043,321555905219, \cdots$

5) $E Q(1,1)=t^{3}-31 t^{2}-25 t-1=0$.

$S E Q 1=3,31,1011,32119,1020995,32454831,1031656755, \cdots$

$\underline{\mathrm{A} 274592]}$

SEQ2 $=3,-25,563,-13297,314947,-7460905,176745971, \cdots \quad$ [Wang

$\underline{\mathrm{A} 248417]}$

6) $E Q(2,0)=t^{3}-83 t^{2}+19 t-1=0$.

$S E Q 1=3,83,6851,567059,46935811,3884905043,321555905219, \cdots$

$S E Q 2=3,19,195,2131,24323,285459,3407043, \cdots$

7) $E Q(0,3)=t^{3}+25 t^{2}-753 t-1=0$.

SEQ1 $=3,-25,2131,-72097,3407043,-139462985,6052005907, \cdots$ 
$S E Q 2=3,-753,567059,-427014249,321555905219,-242142271419073$, $182341168849178195, \cdots$

8) $E Q(1,2)=t^{3}+113 t^{2}+215 t-1=0$.

$S E Q 1=3,-113,12339,-1370009,152158019$, $-16899291873,1876904637555, \cdots$

$S E Q 2=3,215,46451,10011263,2157670723$, $465030524615,100225389595187, \cdots$

9) $E Q(2,1)=t^{3}+289 t^{2}-57 t-1=0$.

SEQ1 $=3,-289,83635,-24186985,6994805571,-2022877384529$, $585010243859443, \cdots$

$S E Q 2=3,-57,3827,-234609,14478659,-893081737,55089756851, \cdots$

10) $E Q(3,0)=t^{3}+753 t^{2}-25 t-1=0$.

$S E Q 1=3,-753,567059,-427014249,321555905219$, $-242142271419073,182341168849178195, \cdots$

SEQ2 $=3,-25,2131,-72097,3407043,-139462985,6052005907, \cdots$

11) $E Q(0,4)=t^{3}-195 t^{2}+6851 t-1=0$.

$S E Q 1=3,195,24323,3407043,497736707,73717030595$, $10964830193411, \cdots$

$S E Q 2=3,6851,46935811,321555905219,2202970354179075$, $15092487193126260931,103398200181210504623875, \cdots$

12) $E Q(1,3)=t^{3}-383 t^{2}-1961 t-1=0$.

$S E Q 1=3,383,150611,58435079,22675983811,8799493140143$, $3414673535363219, \cdots$

$S E Q 2=3,-1961,3844755,-7538813489,14782140708803$, $-28984890560551641,56833708821811482963, \cdots$

13) $E Q(2,2)=t^{3}-1011 t^{2}+563 t-1=0$.

SEQ1 $=3,1011,1020995,1031656755,1042430160131$, $1053316070160371,1064315659783638083, \cdots$

$S E Q 2=3,563,314947,176745971,99189570819,55665038509363$, $31239136201419331, \cdots$

14) $E Q(3,1)=t^{3}-2631 t^{2}-177 t-1=0$.

$S E Q 1=3,2631,6922515,18213602655,47921213873091$, $126083937514694871,331735321674231345363, \cdots$

SEQ2 = 3,-177,26067,-4148169,665643459,-106905033537, $17170878847251, \cdots$

15) $E Q(4,0)=t^{3}-6851 t^{2}+195 t-1=0$.

$S E Q 1=3,6851,46935811,321555905219,2202970354179075$, $15092487193126260931,103398200181210504623875, \cdots$ $S E Q 2=3,195,24323,3407043,497736707,73717030595$, $10964830193411, \cdots$

\section{Beta Sequences of Tangent Values}

In this section we will study the $\beta$ sequences for tangent values. Let $K=\sqrt{7}$ in the formula for $\beta(i, j)$ and $\lambda(i, j)$. Then 


$$
\beta(i, j)=\sum \frac{\sqrt{7} A^{i+j+1}}{B^{i} C^{j}}, \lambda(i, j)=\sum \frac{7 B^{i+1} C^{j+1}}{A^{i+j}} .
$$

\section{Lemma 83. With above notations,}
1) $\beta(0,0)=-7$,
2) $\beta(1,0)=105$,
3) $\beta(0,1)=49$,
4) $\beta(2,0)=-959$,
5) $\beta(0,2)=-119$,
6) $\beta(1,1)=-371$,
7) $\lambda(0,0)=-49$,
8) $\lambda(1,0)=-49$,
9) $\lambda(0,1)=343$,
10) $\lambda(2,0)=-441$,
11) $\lambda(0,2)=-3185$,
12) $\lambda(1,1)=931$.

Proof. To prove (1),

$$
\beta(0,0)=\sqrt{7} \sum A=\sqrt{7} T(1)=-7 .
$$

To prove (2),

$$
\beta(1,0)=\sqrt{7} \sum \frac{A^{2}}{B}=\frac{\sqrt{7}}{F} \sum A B^{3}=\frac{\sqrt{7}}{F} W(1,3)=105 .
$$

To prove (3),

$$
\beta(0,1)=\sqrt{7} \sum \frac{A^{2}}{C}=\frac{\sqrt{7}}{F} \sum A^{3} B=\frac{\sqrt{7}}{F} W(3,1)=49,
$$

To prove (4),

$$
\beta(2,0)=\sqrt{7} \sum \frac{A^{3}}{B^{2}}=\frac{\sqrt{7}}{F^{2}} \sum A^{2} B^{5}=\frac{\sqrt{7}}{F^{2}} W(2,5)=-959,
$$

To prove (5),

$$
\beta(0,2)=\sqrt{7} \sum \frac{A^{3}}{C^{2}}=\frac{\sqrt{7}}{F^{2}} \sum A^{5} B^{2}=\frac{\sqrt{7}}{F^{2}} W(5,2)=-119 .
$$

To prove (6),

$$
\beta(1,1)=\sqrt{7} \sum \frac{A^{3}}{B C}=\frac{\sqrt{7}}{F} \sum A^{4}=\frac{\sqrt{7}}{F} T(4)=-371 .
$$

To prove (7),

$$
\lambda(0,0)=7 \sum B C=7 F \sum \frac{1}{A}=7 F T(-1)=-49 .
$$

To prove (8),

$$
\lambda(1,0)=7 \sum \frac{B^{2} C}{A}=\frac{7}{F} \sum A^{3} B^{2}=\frac{7}{F} W(3,2)=-49 .
$$

To prove (9), 


$$
\lambda(0,1)=7 \sum \frac{B C^{2}}{A}=\frac{7}{F} \sum A^{2} B^{3}=\frac{7}{F} W(2,3)=343 .
$$

To prove (10),

$$
\lambda(2,0)=7 \sum \frac{B^{3} C}{A^{2}}=\frac{7}{F^{2}} \sum A^{5} B^{3}=\frac{7}{F^{2}} W(5,3)=-3185 .
$$

To prove (11),

$$
\lambda(0,2)=7 \sum \frac{B C^{3}}{A^{2}}=\frac{7}{F^{2}} \sum A^{3} B^{5}=\frac{7}{F^{2}} W(3,5)=-441 .
$$

To prove (12),

$$
\lambda(1,1)=7 \sum \frac{B^{2} C^{2}}{A^{2}}=7 F^{2} \sum \frac{1}{A^{4}}=7 F^{2} T(-4)=931 .
$$

Proposition 84. With the above notations,

1) $\beta(n, 0)=-9 \beta(n-1,0)+\beta(n-2,0)+\beta(n-3,0)$,

2) $\beta(0, n)=-\beta(0, n-1)+9 \beta(0, n-2)+\beta(0, n-3)$,

3) $\lambda(n, 0)=-\lambda(n-1,0)+9 \lambda(n-2,0)+\lambda(n-3,0)$,

4) $\lambda(0, n)=a-5 \lambda(0, n-1)+\lambda(0, n-2)+\lambda(0, n-3)$.

Proof. By Proposition 74, $\left\{\frac{A}{B}, \frac{B}{C}, \frac{C}{A}\right\}$ are roots of equation

$$
t^{3}+9 t^{2}-t-1=0
$$

The rest of proof is similar to the proof of Proposition 28.

\section{Proposition 85. With the above notations,}

1) $\beta(n, 0)$ are integers for all integer $n \geq 0$,

2) $\beta(0, n)$ are integers for all integer $n \geq 0$,

3) $\lambda(n, 0)$ are integers for all integer $n \geq 0$,

4) $\lambda(0, n)$ are integers for all integer $n \geq 0$.

Proof. The proof is similar to the proof of Proposition 29

Theorem 86. For integers $i, j$,

$$
\left\{\left(\frac{\sqrt{7} \tan ^{i+j+1} 2 \theta}{\tan ^{i} 4 \theta \tan ^{j} 8 \theta}\right)^{n}+\left(\frac{\sqrt{7} \tan ^{i+j+1} 4 \theta}{\tan ^{i} 8 \theta \tan ^{j} 2 \theta}\right)^{n}+\left(\frac{\sqrt{7} \tan ^{i+j+1} 8 \theta}{\tan ^{i} 2 \theta \tan ^{j} 4 \theta}\right)^{n} \mid n=0,1,2, \cdots\right\}
$$

are integer sequences.

Proof. The proof is similar to the proof of Theorem 30

Remark 87. Note that

$$
\frac{\sqrt{7} A^{i+j+1}}{B^{i} C^{j}} \cdot \frac{\sqrt{7} B^{i+j+1}}{C^{i} A^{j}} \cdot \frac{\sqrt{7} C^{i+j+1}}{A^{i} B^{j}}=-49 .
$$

By Proposition $5\left\{\frac{\sqrt{7} A^{i+j+1}}{B^{i} C^{j}}, \frac{\sqrt{7} B^{i+j+1}}{C^{i} A^{j}}, \frac{\sqrt{7} C^{i+j+1}}{A^{i} B^{j}}\right\}$ are the roots of a monic equation

$$
E Q(i, j)=t^{3}-\beta(i, j) t^{2}+\lambda(i, j) t+49=0 .
$$

Let $\operatorname{SEQ}(i, j)$ be the associated sequence. 
Example 88. List of associated sequences:

1) $E Q(0,0)=t^{3}+7 t^{2}-49 t+49=0$.

$S E Q=3,-7,147,-1519,18179,-208887,2427411, \cdots \quad$ [Wang A275195]

2) $E Q(0,1)=t^{3}-49 t^{2}+343 t+49=0$.

$S E Q=3,49,1715,67081,2696323,109027009,4414197683, \cdots$

3) $E Q(1,0)=t^{3}-105 t^{2}-49 t+49=0$.

$S E Q=3,105,11123,1172913,123695747,13044981145,1375726639091, \cdots$

4) $E Q(0,2)=t^{3}+119 t^{2}-3185 t+49=0$.

$S E Q=3,-119,20531,-2822351,401256835,-56739757319$,

$8030172435635, \cdots$

5) $E Q(1,1)=t^{3}+371 t^{2}+931 t+49=0$.

$S E Q=3,-371,135779,-50028755,18434276035$,

$-6792546291251,2502874814474531, \cdots$

6) $E Q(2,0)=t^{3}+959 t^{2}-441 t+49=0$.

$S E Q=3,-959,920563,-883242983,847436035971$,

$-813080713759279,780118167065917939, \cdots$

7) $E Q(0,3)=t^{3}-553 t^{2}+28959 t+49=0$.

$S E Q=3,553,247891,121069249,59772592131$, $29548186919993,14609186938841299, \cdots$

8) $E Q(1,2)=t^{3}-1281 t^{2}-8281 t+49=0$.

$S E Q=3,1281,1657523,2133894777,2747245094531$,

$3536891667523921,4553508058165109939, \cdots$

9) $E Q(2,1)=t^{3}-3353 t^{2}+2303 t+49=0$.

$S E Q=3,3353,11238003,37673301953,126292700163203$, $423372661482159753,1419277680015213999603, \cdots$

10) $E Q(3,0)=t^{3}-8729 t^{2}-49 t+49=0$.

$S E Q=3,8729,76195539,665111287505,5805760161784835$, $50678513038939331049,442373024766558895095891, \cdots$

11) $E Q(0,4)=t^{3}+1575 t^{2}-263473 t+49=0$.

$S E Q=3,-1575,3007571,-5151894447,8906647585283$,

$-15385355179826135,26578595819906258387, \cdots$

12) $E Q(1,3)=t^{3}+4515 t^{2}+75411 t+49=0$.

$S E Q=3,-4515,20234403,-91017849027,409419692013507$,

$-1841666163419494755,8284247983904462844771, \cdots$

13) $E Q(2,2)=t^{3}+11711 t^{2}-21609 t+49=0$.

$S E Q=3,-11711,137190739,-1606893807575,18821297935763715$, $-220450943500739100751,2582107708842987323583571, \cdots$

14) $E Q(3,1)=t^{3}+30499 t^{2}+6419 t+49=0$.

$S E Q=3,-30499,930176163,-28369247022403,865227694136973251$, $-26388397341332489009379,804814176618121010169849699, \cdots$

15) $E Q(4,0)=t^{3}+79415 t^{2}-3969 t+49=0$.

$S E Q=3,-79415,2011782867,-159766051581087$, $12687828971082114563,-1007604571850043430715431$, $80018967431472213811706126675, \cdots$ 


\section{Gamma Sequences of Tangent Values}

In this section we will study the $\gamma$ sequences for tangent values. Let $K=\sqrt{7}$ in the formula for $\gamma(i, j)$ and $\mu(i, j)$. Then

$$
\gamma(i, j)=\sum \frac{\sqrt{7} A^{i+j-1}}{B^{i} C^{j}}, \mu(i, j)=\sum \frac{7 B^{i-1} C^{j-1}}{A^{i+j}} .
$$

Lemma 89. With the above notations,
1) $\gamma(0,0)=7$,
2) $\gamma(1,0)=7$
3) $\gamma(0,1)=7$,
4) $\gamma(2,0)=-49$,
5) $\gamma(0,2)=7$,
6) $\gamma(1,1)=-21$,
7) $\mu(0,0)=7$,
8) $\mu(1,0)=7$,
9) $\mu(0,1)=7$,
10) $\mu(2,0)=-49$,
11) $\mu(0,2)=-105$,
12) $\mu(1,1)=35$.

Proof. The proof is similar to the proof of Lemma 33.

\section{Proposition 90. With the above notations,}
1) $\gamma(n, 0)=-9 \gamma(n-1,0)+\gamma(n-2,0)+\gamma(n-3,0)$,
2) $\gamma(0, n)=-\gamma(0, n-1)+9 \gamma(0, n-2)+\gamma(0, n-3)$,
3) $\mu(n, 0)=-\mu(n-1,0)+9 \mu(n-2,0)+\mu(n-3,0)$,
4) $\mu(0, n)=-9 \mu(0, n-1)+\mu(0, n-2)+\mu(0, n-3)$.

Proof. The proof is similar to the proof of Proposition 34.

Proposition 91. With the above notations,

1) $\gamma(n, 0)$ are integers for all integers $n \geq 0$.

2) $\gamma(0, n)$ are integers for all integers $n \geq 0$.

3) $\mu(n, 0)$ are integers for all integers $n \geq 0$.

4) $\mu(0, n)$ are integers for all integers $n \geq 0$.

Proof. The proof is similar to the proof of Proposition 35.

Theorem 92. For integers $i, j$,

$$
\left\{\left\{\left(\frac{\sqrt{7} \tan ^{i+j-1} 2 \theta}{\tan ^{i} 4 \theta \tan ^{j} 8 \theta}\right)^{n}+\left(\frac{\sqrt{7} \tan ^{i+j-1} 4 \theta}{\tan ^{i} 8 \theta \tan ^{j} 2 \theta}\right)^{n}+\left(\frac{\sqrt{7} \tan ^{i+j-1} 8 \theta}{\tan ^{i} 2 \theta \tan ^{j} 4 \theta}\right)^{n} \mid n=0,1,2, \cdots\right\}\right.
$$

is integer sequences.

Proof. The proof is similar to the proof of Theorem 36.

Remark 93. Note that

$$
\frac{\sqrt{7} A^{i+j-1}}{B^{i} C^{j}} \cdot \frac{\sqrt{7} B^{i+j-1}}{C^{i} A^{j}} \cdot \frac{\sqrt{7} C^{i+j-1}}{A^{i} B^{j}}=-7 .
$$

By Proposition $5\left\{\frac{\sqrt{7} A^{i+j-1}}{B^{i} C^{j}}, \frac{\sqrt{7} B^{i+j-1}}{C^{i} A^{j}}, \frac{\sqrt{7} C^{i+j-1}}{A^{i} B^{j}}\right\}$ are the roots of a monic 
equation

$$
E Q(i, j)=t^{3}-\gamma(i, j) t^{2}+\mu(i, j) t+7=0 .
$$

Let $\operatorname{SEQ}(i, j)$ be the associated sequence.

Example 94. List of associated sequences.

1) $E Q(0,0)=t^{3}-7 t^{2}+7 t+7=0$.

$S E Q=3,7,35,175,931,5047,27587, \cdots \quad$ [Witula 215575]

2) $E Q(0,1)=t^{3}-7 t^{2}+7 t+7=0$.

$S E Q=3,7,35,175,931,5047,27587, \cdots$

3) $E Q(1,0)=t^{3}-7 t^{2}+7 t+7=0$.

$S E Q=3,7,35,175,931,5047,27587, \cdots$

4) $E Q(0,2)=t^{3}-7 t^{2}-105 t+7=0$.

$S E Q=3,7,259,2527,44835,577367,8731555, \cdots$

5) $E Q(1,1)=t^{3}+21 t^{2}+35 t+7=0$.

$S E Q=3,-21,371,-7077,135779,-2606261,50028755, \cdots$

[Delham A108716]

6) $E Q(2,0)=t^{3}+49 t^{2}-49 t+7=0$.

$S E Q=3,-49,2499,-124873,6241571,-311973249,15593400291, \cdots$

7) $E Q(0,3)=t^{3}-63 t^{2}+959 t+7=0$.

$S E Q=3,63,2051,68775,2365475,83055343,2963514659, \cdots$

8) $E Q(1,2)=t^{3}-63 t^{2}-273 t+7=0$.

$S E Q=3,63,4515,301623,20234403,1357078863,91017849027, \cdots$

9) $E Q(2,1)=t^{3}-175 t^{2}+63 t+7=0$.

$S E Q=3,175,30499,5326279,930176163,162445059455,28369247022403, \cdots$

10) $E Q(3,0)=t^{3}-455 t^{2}+119 t+7=0$.

$S E Q=3,455,206787,94033919,42760822307$,

$19444982665815,8842377916853859, \cdots$

11) $E Q(0,4)=t^{3}-7 t^{2}-8729 t+7=0$.

$S E Q=3,7,17507,183631,154103971,2681520247,1363942919171, \cdots$

12) $E Q(1,3)=t^{3}+245 t^{2}+2499 t+7=0$.

$S E Q=3,-245,55027,-12869381,3015487587$, $-706634260885,165589780522579, \cdots$

13) $E Q(2,2)=t^{3}+609 t^{2}-721 t+7=0$.

$S E Q=3,-609,372323,-227183817,138623393699$, $-84585448901009,51612487437858179, \cdots$

14) $E Q(3,1)=t^{3}+1589 t^{2}+259 t+7=0$.

$S E Q=3,-1589,2524403,-4010864837,6372610416739$, $-10125039155876309,16087036740665573459, \cdots$

15) $E Q(4,0)=t^{3}+4137 t^{2}-553 t+7=0$.

$S E Q=3,-4137,17115875,-70810662657,292953176519843$, $-1211986449678850937,5014149945923696438147, \cdots$

\section{Conflicts of Interest}

The author declares no conflicts of interest regarding the publication of this paper. 


\section{References}

[1] Witula, R. (2009) Type Trigonometric Formulas: The General Form for the Argument $2 \pi / 7$. Journal of Integer Sequences, 12, 1-23.

[2] Berndt, B.C. and Zhang, L.C. (1992) Ramanujans Identities for Eta-Functions. Mathematische Annalen, 292, 561-573 https://doi.org/10.1007/BF01444636

[3] Wang, K. (2016) Sums of Mixed Trigonometric Powers.

[4] Bankoff, L. and Garfunkel, J. (1973) The Heptagonal Triangle. Mathematics Magazine, 46, 7-19. https://doi.org/10.1080/0025570X.1973.11976267

[5] WIKI. Heptagonal Triangle. https://en.wikipedia.org/wiki/Heptagonal_triangle

[6] OIES The On-Line Encyclopedia of Integer Sequences. https://oeis.org/wiki/Welcome

\section{List of Integer Sequences}

List of integer sequences from OEIS [6]:

1) Adamson G. Adamson A116423, A180262, A094430.

2) Adamson-Bagula G. Adamson and Roger L. Bagula, A120757, A122161, A122600.

3) Bagula R. Bagula, A106803, A152046.

4) Barry P. Barry, A094648, A096975, A096976.

5) Bernstein-Sloane-Wilson M. Bernstein, N. Sloane, R. Wilson, A000975.

6) Butler S. Butler A136776.

7) Delham P. Delham, $\underline{\text { A085810 }}, \underline{\text { A199853 }}, \underline{\text { A108716. }}$

8) Deutsch E. Deutsch A109110.

9) Encyclopedia (AT) pommard.inria.fr encyclopedia(AT)pommard.inria.fr, A052534, A052547.

10) Hanna P. Hanna, A136752.

11) Kimberling C. Kimberling, A287381.

12) W. Lang, A181879.

13) Librandi V. Librandi, A271945, A271944.

14) Pharo A. Pharo, A219788.

15) Sloane N. Sloane, A077925, A001045, A006053, A078038, A006054, $\underline{\mathrm{A} 006356}, \underline{\mathrm{A} 077998}, \underline{\mathrm{A} 033304}, \underline{\mathrm{A} 028495}$.

16) Wang Kai Wang A274032, $\underline{\mathrm{A} 274075}, \underline{\mathrm{A} 274220}, \underline{\mathrm{A} 274592}, \underline{\mathrm{A} 248417}$ $\underline{\mathrm{A} 274663}, \underline{\mathrm{A} 274664}, \underline{\mathrm{A} 274975}, \underline{\mathrm{A} 275195}, \underline{\mathrm{A} 275830}, \underline{\mathrm{A} 275831}, \underline{\mathrm{A} 287396}, \underline{\mathrm{A} 287405}$, A320918.

17) Witula R. Witula, A214683, A215007, A215008, A215076, A215112, A215139, A006054, A215575, A215100, A215575, A215560, A215569, A215666, $\mathrm{A} 215794, \mathrm{~A} 215829, \mathrm{~A} 215404, \mathrm{~A} 217274$.

18) Wieder T. Wieder, A109509. 